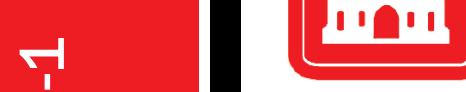

US Army Corps of Engineers $s_{\circledast}$

Engineer Research and

Development Center

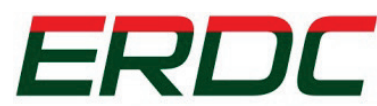

INNOVATIVE SOLUTIONS for a safer, better world

Dredging Operations and Environmental Research Program

\title{
The Feasibility of Performing Particle- Tracking-Based Flow Measurements with Acoustic Cameras
}

David L. Young and Brian C. McFall

August 2017

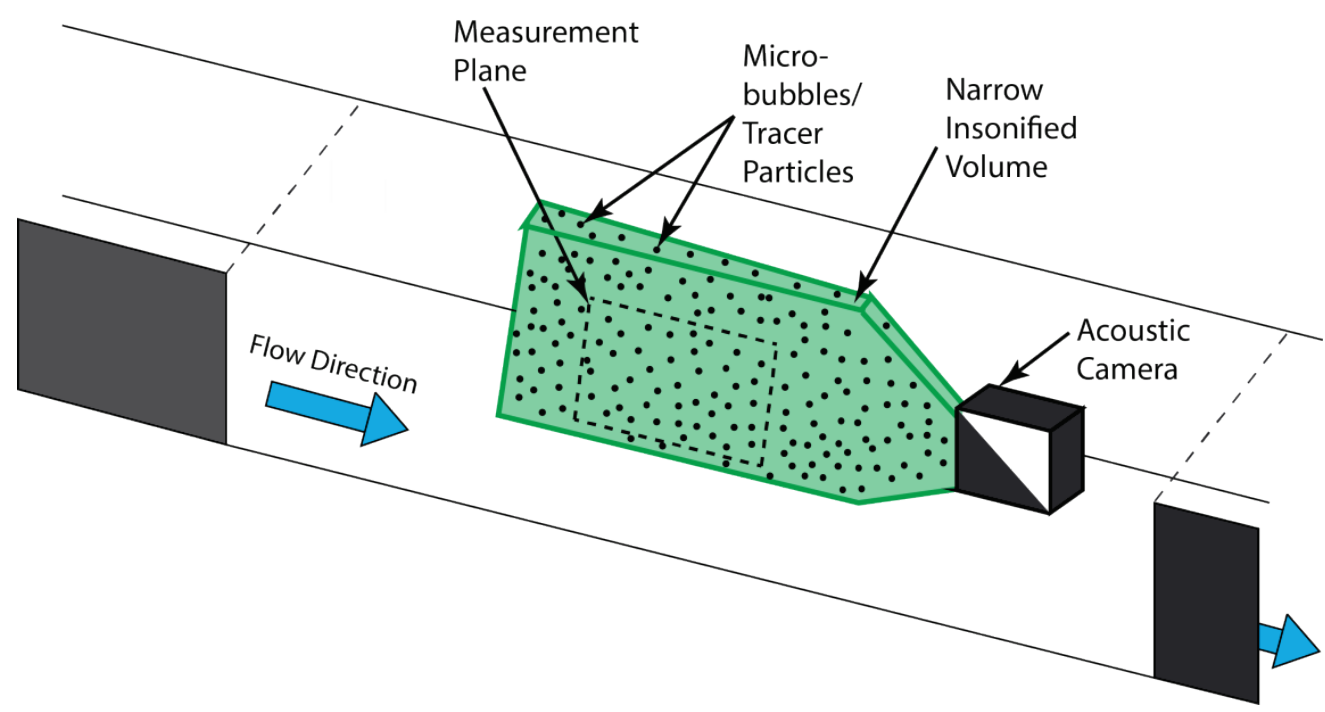

Approved for public release; distribution is unlimited. 
The U.S. Army Engineer Research and Development Center (ERDC) solves the nation's toughest engineering and environmental challenges. ERDC develops innovative solutions in civil and military engineering, geospatial sciences, water resources, and environmental sciences for the Army, the Department of Defense, civilian agencies, and our nation's public good. Find out more at www.erdc.usace.army.mil.

To search for other technical reports published by ERDC, visit the ERDC online library at http://acwc.sdp.sirsi.net/client/default. 


\section{The Feasibility of Performing Particle- Tracking-Based Flow Measurements with Acoustic Cameras}

David L. Young and Brian C. McFall

Coastal and Hydraulics Laboratory

U.S. Army Engineer Research and Development Center

3909 Halls Ferry Road

Vicksburg, MS 39180-6199

Final report

Approved for public release; distribution is unlimited.

Prepared for U.S. Army Corps of Engineers

Washington, DC 20314-1000

Under DOER Project 17-02, "The Feasibility of Performing Tracking-Based Blow Measurements with Acoustic Cameras" 


\section{Abstract}

Modern science lacks the capability to quantify flow velocity fields in turbid environments, particularly in the field. Existing optical-camera particle-tracking-based techniques developed to quantify two-dimensional (2D) velocity fields in laboratory environments, such as particle image velocimetry (PIV), are equipment intensive and can only be performed in a reasonably transparent fluid (so the camera can observe the light reflected by the particles). Acoustic-based flow measurement equipment used in the field (e.g., acoustic Doppler velocimeter and acoustic Doppler current profiler) can readily acquire velocities in turbid flows but only measure point velocities or velocities along a transect. Consequently, they do not provide the capability to quantify spatial gradients of velocity when deployed individually. The objective of this report is to explore whether acoustic cameras can be used in place of optical cameras in particletracking-based flow measurement techniques, such as PIV. Acoustic cameras may offer the capability to track a large number of particles in a plane as is required for traditional PIV and are designed to operate in turbid environments. The development of an acoustic camera particletracking-based flow measurement system will be able to quantify $2 \mathrm{D}$ flow velocity fields in turbid environments and be readily adaptable to a fieldbased system.

DISCLAIMER: The contents of this report are not to be used for advertising, publication, or promotional purposes. Citation of trade names does not constitute an official endorsement or approval of the use of such commercial products. All product names and trademarks cited are the property of their respective owners. The findings of this report are not to be construed as an official Department of the Army position unless so designated by other authorized documents. 


\section{Contents}

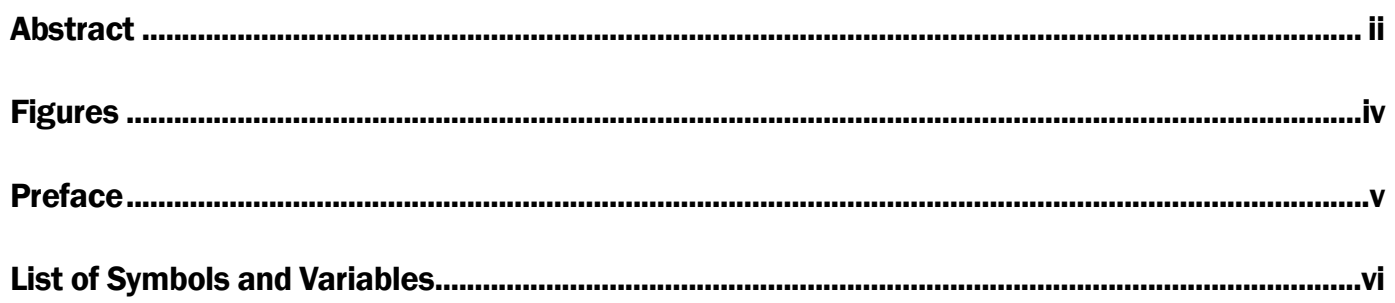

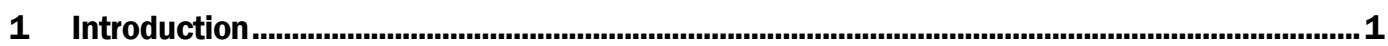

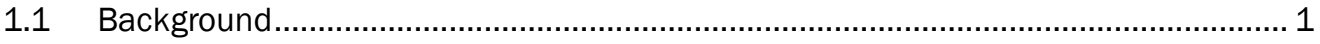

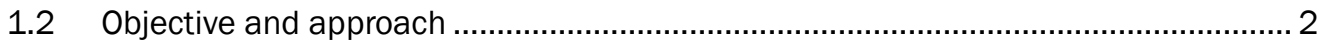

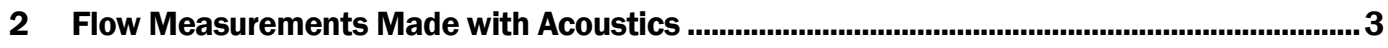

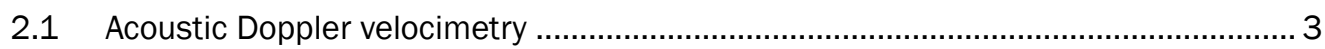

2.1.1 Commercial acoustic Doppler velocimeters (ADVs) ............................................ 6

2.1.2 Lagrangian particle velocities ............................................................................. 8

2.2 Particle tracking with acoustics.................................................................... 8

2.2.1 Tracking single particles with transducers ......................................................... 8

2.2.2 Particle tracking with an acoustic camera ........................................................ 10

3 The Possibility of Particle Tracking Velocimetry (PTV) and Particle Image

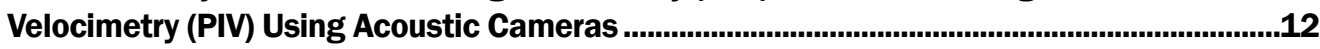

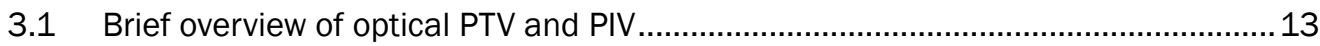

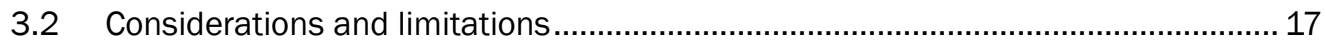

3.2.1 Acoustic-camera resolution and frame rate ........................................................ 17

3.2.2 Particle dynamics and ramifications for PTV/PIV ............................................... 19

3.2.3 Plane wave scattering from particles.................................................................. 23

3.2.4 Out-of-plane particles and out-of-plane fluid motion ........................................... 26

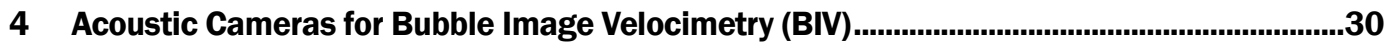

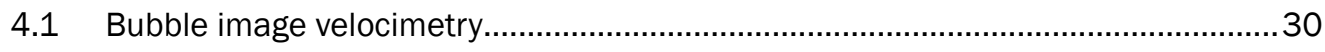

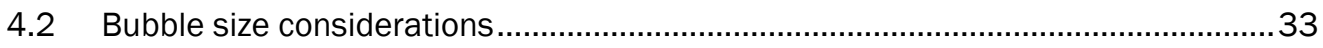

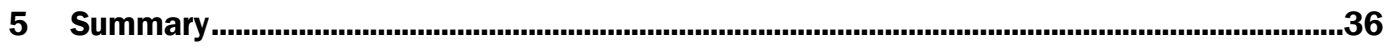

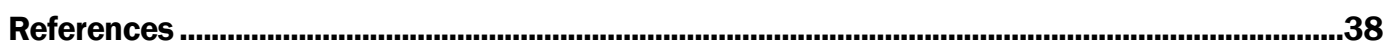

Report Documentation Page 


\section{Figures}

\section{Figures}

Figure 2-1. Schematic detailing the transducer configuration to acquire 3D velocity measurements using acoustic Doppler velocimetry described in Mordant et al. (2005). The propagation direction of the sound waves emitted by the emitting transducer is given by kinc. Figure reproduced with minor edits from Mordant et al. (2005) with the permission of AIP Publishing.

Figure 2-2. Schematic detailing the transducer configuration to acquire 3D velocity measurements using acoustic Doppler velocimetry described in Gervais et al. (2007). Figure reproduced from Gervais et al. (2007) with minor edits with the permission of Springer-Verlag.

Figure 2-3. Schematic detailing the transducer configuration to acquire 3D velocity measurements using acoustic Doppler velocimetry described in Kraus et al. (1994). Figure adapted from Kraus et al. (1994) with permission from ASCE.

Figure 2-4. Image of a Nortek Vector Velocimeter (Nortek AS, Rud, Norway). Reprinted from http://www.nortek-as.com/en/products/velocimeters/ with permission.

Figure 2-5. Schematic detailing the setup of the Wu et al. (2008) experiment. Figure reproduced from Wu et al. (2008) with the permission of AIP Publishing.

Figure 3-1. Schematic detailing a traditional optical PIV experimental setup.

Figure 3-2. (a) Relative seeding density to track aggregate motion of particles in a subwindow (PIV). (b) Relative particle seeding density required to track individual particles (PTV). Note that the seeding density is far less for PTV, such that individual particles can be tracked between subsequent images.

Figure 3-3. Image of a Sound Metrics ARIS Explorer 3000 Imaging Sonar (Sound Metrics Corp., Bellevue, WA). Reprinted from www.soundmetrics.com/Products/ARIS-

Sonars/ARIS-Explorer-3000 with permission.

Figure3-4. Diagram of the plan view and the side view of the measurement volume of a Sound Metrics ARIS Explorer 3000 Imaging Sonar.....

Figure 4-1. Sample image of a plunging breaking wave impacting on the model structure in Ryu et al. (2005). The region boxed in green delineates a highly aerated region in which BIV must be used to obtain velocity measurements. Figure reprinted from Ryu et al. (2005); IOP Publishing. Reproduced with permission. All rights reserved.

Figure 4-2. Mean velocity field of the plunging breaking wave impinging on the model structure measured with BIV (Ryu et al. 2005). Note the measurement area is confined to the high void fraction region of the flow. Figure reprinted from Ryu et al. (2005); IOP Publishing. Reproduced with permission. All rights reserved. 


\section{Preface}

This study was conducted for Headquarters, U.S. Army Corps of Engineers (HQUSACE), Washington, DC, under the USACE Dredging Operations and Environmental Research (DOER) Program, Project 17-02, "The Feasibility of Performing Particle Tracking-based Flow Measurements with Acoustic Cameras." The HQUSACE DOER Program Manager was Dr. Todd S. Bridges, U.S. Army Engineer Research and Development Center (ERDC), Environmental Laboratory (EL). Mr. Jeffrey A. McKee was the HQUSACE Navigation Business Line Manager overseeing the DOER Program. Mr. W. Jeff Lillycrop, ERDC Coastal and Hydraulics Laboratory (CHL), was the ERDC Technical Director for Civil Works and Navigation Research, Development, and Technology Transfer (RD\&T) portfolio.

The work was performed by the ERDC-CHL Estuarine Engineering Branch (HFE) and Coastal Engineering Branch (HNC) of the Flood and Storm Protection Division (HF) and the Navigation Division (HN), respectively. At the time of publication, Dr. Drew A. Loney was acting Chief, CEERDHFE; Ms. Tanya M. Beck was Chief, CEERD-HN-C; Dr. Cary A. Talbot was Chief, CEERD-HF, and Dr. Jackie S. Pettway was Chief, CEERD-HN. The ERDC-CHL Deputy Director was Mr. Jeffrey R. Eckstein, and the Director was Mr. José E. Sánchez.

The Commander of ERDC was COL Bryan S. Green, and the Director was Dr. David W. Pittman. 


\section{List of Symbols and Variables}

\begin{tabular}{|c|c|c|}
\hline Variable & Definition & Example Units \\
\hline$f$ & Frequency & Hertz $(\mathrm{Hz})$ \\
\hline$\hat{n}$ & Direction vector & Meters $(m)$ \\
\hline$\hat{V}$ & Velocity vector & Meters per second $(\mathrm{m} / \mathrm{s})$ \\
\hline$c$ & Speed of sound in fluid & Meters per second $(\mathrm{m} / \mathrm{s})$ \\
\hline$\phi$ & Phase shift & Radians (Rad) \\
\hline$\omega$ & Angular frequency & Radians per second $(\mathrm{Rad} / \mathrm{s})$ \\
\hline$s$ & Spacing between transducers & Meters $(m)$ \\
\hline$\theta$ & Angle & Radians (Rad) \\
\hline$R e$ & Reynolds number & - \\
\hline$U$ & Free-stream velocity & Meters per second $(\mathrm{m} / \mathrm{s})$ \\
\hline$d$ & Diameter & Millimeters $(\mathrm{mm})$ \\
\hline$v$ & Kinematic viscosity & Meters squared per second $\left(\mathrm{m}^{2} / \mathrm{s}\right)$ \\
\hline$V_{p}$ & Particle (spherical) velocity & Meters per second $(\mathrm{m} / \mathrm{s})$ \\
\hline$\rho_{s}$ & Particle (spherical) density & Kilograms per meter cubed $\left(\mathrm{kg} / \mathrm{m}^{3}\right)$ \\
\hline$\rho$ & Fluid density & Kilograms per meter cubed $\left(\mathrm{kg} / \mathrm{m}^{3}\right)$ \\
\hline$\mu$ & Dynamic viscosity & Kilograms per meter second $(\mathrm{kg} / \mathrm{m} \cdot \mathrm{s})$ \\
\hline$\tau_{p}$ & Characteristic response time of spherical particle & Seconds $(s)$ \\
\hline$\tau_{K}$ & Kolmogorov time scale & Seconds $(s)$ \\
\hline$L$ & Flow characteristic length scale & Meters $(m)$ \\
\hline$\tau_{f}$ & Characteristic time scale of the flow & Seconds $(s)$ \\
\hline Stk & Stokes number & - \\
\hline$r$ & Distance away from spherical particle & Meters $(m)$ \\
\hline$\lambda$ & Wavelength & Meters $(m)$ \\
\hline$\Delta z_{0}$ & Thickness of laser sheet & Millimeters $(\mathrm{mm})$ \\
\hline
\end{tabular}




\section{Introduction}

\subsection{Background}

Many natural flows are turbid, such as lake circulation as well as riverine, estuarine, and coastal flows, which frequently contain high suspended sediment concentrations. Despite the ubiquity of turbid flows, modern flow measurement lacks the capability to acquire velocity vector fields in highly turbid flows. Measurements of the velocity field are required to compute spatial gradients of the velocity and other related characteristics such as the strain rate and vorticity-critical features for describing more complex flows.

There are several techniques used to acquire velocity vector fields in controlled laboratory settings, and most rely on using optical cameras to track the motion of a controlled amount of tracer particles suspended in an otherwise clear fluid. Two-dimensional (2D) velocity vector fields have been quantifiable with these methods since the late 1980s (Adrian 1986), and more recent techniques are able to measure fully three-dimensional (3D) vector fields (Elsinga et al. 2005, 2006a, 2006b). However, the very definition of turbidity renders optical-camera-based particle-tracking techniques ineffective in the necessarily opaque turbid flows. Conversely, acoustic-based flow measurement techniques are quite capable of making velocity measurements in turbid flows (acoustic Doppler velocimeters [ADVs] and acoustic Doppler current profilers [ADCPs] actually require sufficiently high suspended sediment concentrations to operate) but are not able to measure the velocity field in a 2D plane as required to compute any component of vorticity and the majority of the strain rate tensor components.

It may be possible to combine the attractive attributes of laboratory optical-camera-based particle-tracking techniques (measurements of 2D velocity vector fields) and acoustic-based flow measurement techniques (ability to obtain velocities in turbid flows) by using an acoustic camera to track the suspended particles. Acoustic cameras can easily operate in turbid flows (Belcher et al. 2001; Kim et al. 2005; Belcher 2006; Wu et al. 2008) and locate the position of objects in two dimensions, as required for particle-tracking-based flow measurement. However, the use of acoustic cameras for particle-tracking-based flow measurement is a heretofore 
uninvestigated area of experimental flow measurement, and only a single study (Wu et al. 2008) assesses the feasibility of acoustic cameras for any particle-tracking application. Consequently, several steps will need to be taken to close the gap between generic particle tracking with acoustic cameras and the particle tracking required for flow measurement.

\subsection{Objective and approach}

This report discusses the potential of performing particle-tracking-based flow measurement using an acoustic camera to obtain velocity vector fields in turbid flows. This report presents a review of several acoustic-based flow measurement techniques and working principles, as well as the operating principles of acoustic cameras specifically. Subsequently, there is a discussion of three specific types of particle-tracking-based, flow measurement techniques (particle tracking velocimetry [PTV], particle image velocimetry [PIV], and bubble image velocimetry [BIV]). Specifically, there is a description of how they are typically performed in laboratory settings and how acoustic cameras may be used in place of optical cameras in these techniques, as well as any considerations that may need to be addressed in doing so. If successfully implemented, an acousticcamera, particle-tracking-based flow measurement system will offer the capability to quantify 2D flow velocity fields in turbid environments and will be substantially easier to adapt into a field-ready system than opticalcamera-based techniques. 


\section{Flow Measurements Made with Acoustics}

Before delving into using acoustic cameras to track suspended particles and its potential application to particle tracking and particle image velocimetry, it is important to review acoustic-based flow measurement techniques. This allows better understanding of the physics underpinning acoustic cameras and the gaps in existing acoustic measurement capability that may be filled by an acoustic-camera, particle-tracking-based technique. The following sections describe acoustic Doppler velocimetryboth the field as well as the commercially available instruments that bear the name. More spatially and temporally resolved acoustic-based techniques to track Lagrangian particle velocities and particle motion will then be reviewed, followed by a description of acoustic cameras and a brief review of a study evaluating their use for particle tracking.

\subsection{Acoustic Doppler velocimetry}

In the context of hydraulics, acoustic Doppler velocimetry is a technique by which the velocity of a particle (or particles) suspended in a fluid flow is estimated by measuring the Doppler shift in frequency of a sound wave before and after it scatters off the particle. In many cases, these particles are assumed to faithfully follow the fluid motion; thus the estimated particle velocity is a surrogate for the flow velocity. The theory and technology required to make these measurements has existed for some time (Kraus et al. 1994), and instruments that do so are commercially available (e.g., Nortek Vector ADV-Nortek AS).

The underpinnings for a device to measure a single velocity component using acoustic Doppler velocimetry are as follows. Directionally focused ultrasonic sound waves with frequency $f_{i}$ are emitted from a transducer along a vector $\hat{n}_{i}$ (Gervais et al. 2007). These sound waves are scattered off tracer particles suspended in the flow, and due to the Doppler effect, the scattered sound will have a different frequency $f_{r}$. A second receiving transducer receives the scattered sound waves from a different direction than the incoming sound wave $\left(\hat{n}_{r}\right)$. The frequencies of the incident $\left(f_{i}\right)$ and scattered $\left(f_{r}\right)$ sounds are then related by the following expression (Gervais et al. 2007):

$$
\frac{f_{i}-f_{r}}{f_{i}}=\frac{\widehat{V}}{c}
$$


where $c$ is the speed of sound in the fluid and $\widehat{V}$ is the velocity along the direction defined by $\hat{n}_{i}-\hat{n}_{r}$.

It is possible to measure all three components of the velocity by adding additional transducers, and several configurations to accomplish this are found in the literature. Mordant et al. $(2004,2005)$ used a single emitting transducer and two orthogonal receiving transducer arrays-each transducer array contained nine receiving transducers arrayed in the shape of a cross (Figure 2-1). This configuration is able to quantify both the $3 \mathrm{D}$ velocity of the particle and its position within the measurement volume (the intersection of the emitting and receiving transducer measurement regions-the shaded region in Figure 2-1). Gervais et al. (2007) used two emitting/receiving transducer pairs to measure particle velocity-one pair above and one below the measurement volume (Figure 2-2). Each emitting transducer operates at a different frequency, and the scattered sound from each emitting transducer is picked up by each receiving transducer, yielding the tracer velocity along four nonorthogonal directions $(i, j, k, l)$. Using a matrix transformation, these velocities can be manipulated to determine the velocities in standard orthogonal Cartesian coordinate directions $(x, y, z)$. However, the most well-known configuration is that employed by most commercially available ADVs (e.g., SonTek ADV, SonTek; Nortek Vector ADV, Nortek AS), following the recommendations of Kraus et al. (1994). In this configuration, the emitting transducer is placed directly above the measurement volume, and three receiving transducers are equidistantly spaced $\left(120^{\circ}\right.$ apart) around the emitting transducer (Figure 2-3). The receiving transducers are offset from the emitting transducer such that the scattered sound returns to the receiving transducers at some angle to the incident sound ( $30^{\circ}$ in Kraus et al. [1994]). 
Figure 2-1. Schematic detailing the transducer configuration to acquire 3D velocity measurements using acoustic Doppler velocimetry described in Mordant et al. (2005). The propagation direction of the sound waves emitted by the emitting transducer is given by $\boldsymbol{k}_{\text {inc }}$. Figure reproduced with minor edits from Mordant et al. (2005) with the permission of AIP Publishing.

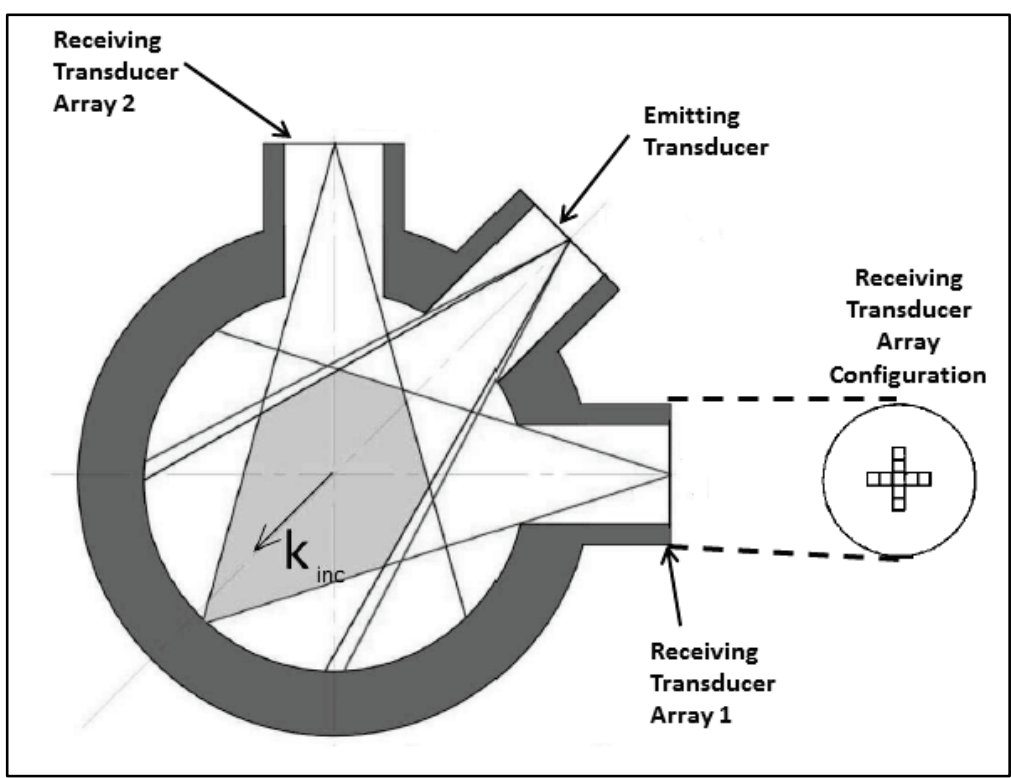

Figure 2-2. Schematic detailing the transducer configuration to acquire $3 \mathrm{D}$ velocity measurements using acoustic Doppler velocimetry described in Gervais et al. (2007). Figure reproduced from Gervais et al. (2007) with minor edits with the permission of Springer-Verlag.

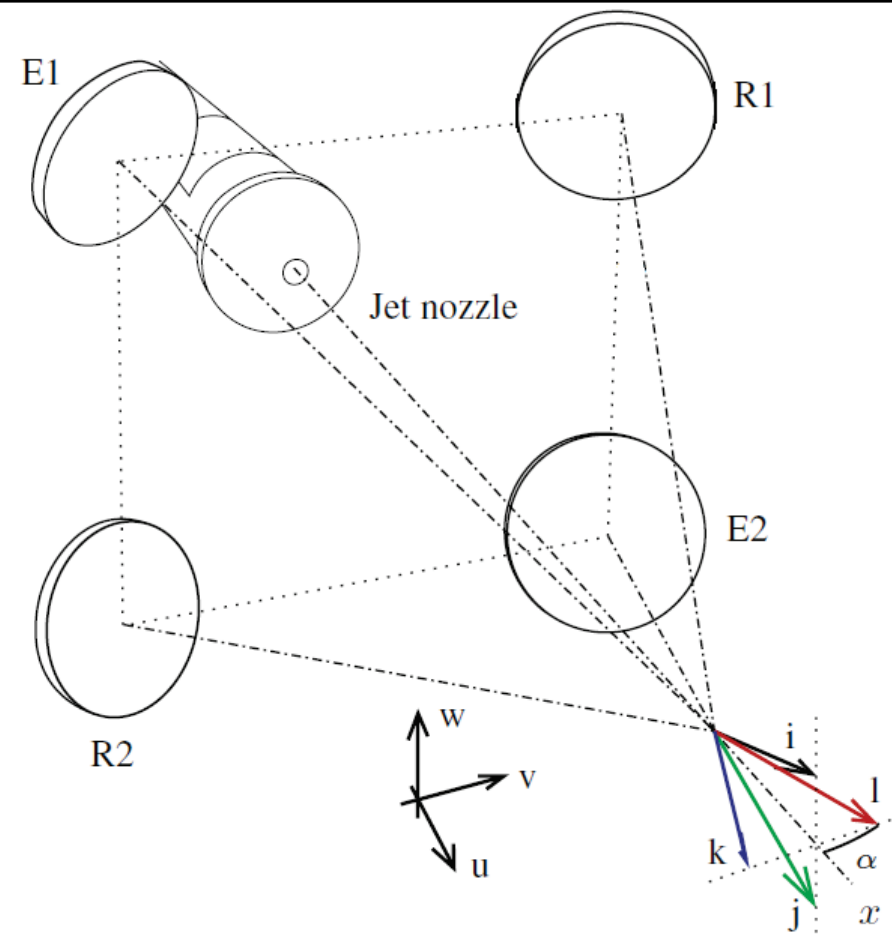


Figure 2-3. Schematic detailing the transducer configuration to acquire 3D velocity measurements using acoustic Doppler velocimetry described in

Kraus et al. (1994). Figure adapted from Kraus et al. (1994) with permission from ASCE.

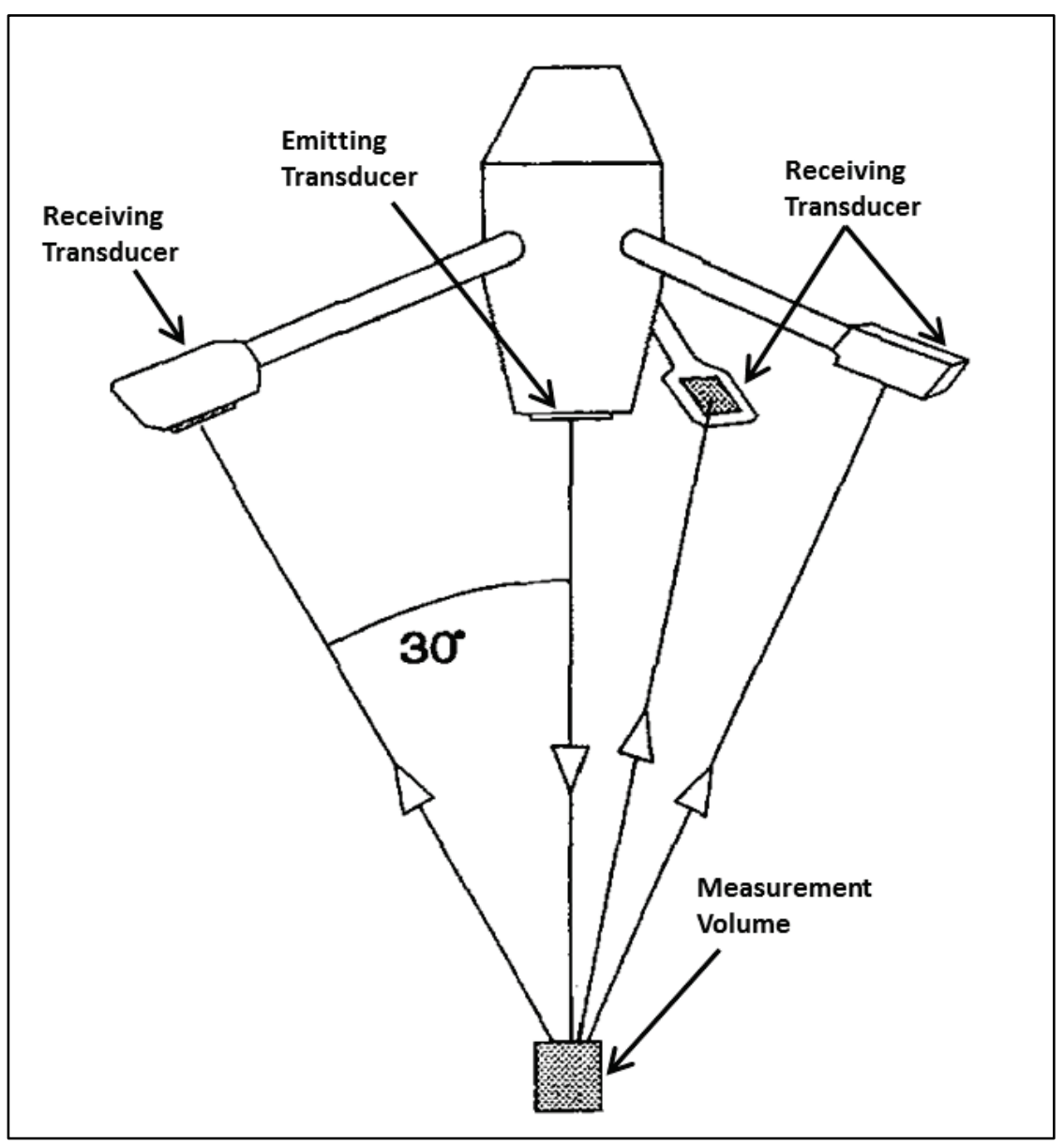

\subsubsection{Commercial acoustic Doppler velocimeters (ADVs)}

The commercially available ADVs (e.g., Nortek Vector Velocimeter, Nortek AS-Figure 2-4) essentially measure the average velocity of all the particles suspended in a small measurement volume, yielding velocity measurements with relatively high degrees of spatial and temporal resolution. Additionally, they offer many advantages over alternative velocity measurement devices, particularly for large-scale hydraulic models or field data collection. ADVs are small, robust, and relatively unaffected by biofouling. Furthermore, the sampling technique is nonintrusive and yields $3 \mathrm{D}$ velocity vectors. In addition, the sampling rate is sufficiently fast and the sampling volume sufficiently small to resolve finer-scale turbulent velocity characteristics, such as the Reynolds stress, turbulence intensity, etc. Finally, the small measurement volume allows for measurements closer to the bed than possible with devices such as 
ADCPs or time-travel acoustic sensors-a key feature when measuring turbulent boundary layers. Consequently, they have been used to measure a wide array of flows, from laboratory experiments of the Reynolds stress in a turbulent boundary layer at different heights above the bed (Voulgaris and Trowbridge 1998) to field measurements of flow through saltmarsh vegetation (Young et al. 2016).

Figure 2-4. Image of a Nortek Vector Velocimeter (Nortek AS, Rud, Norway). Reprinted from http://www.nortekas.com/en/products/velocimeters/ with permission.

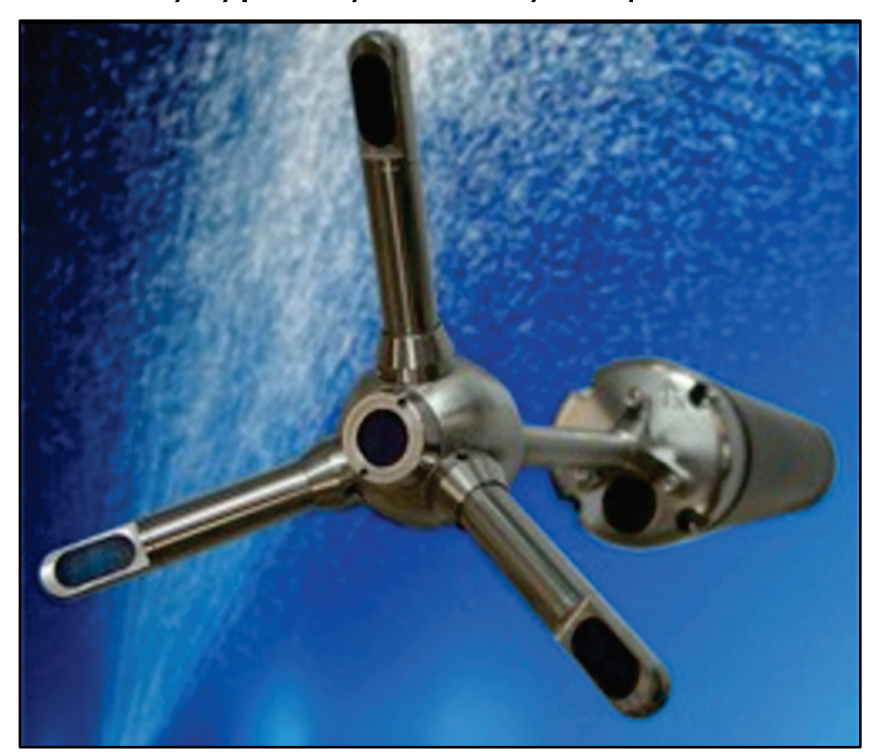

However, several factors must be considered before using ADVs to measure certain types of flows. When measuring turbulent flows, great care must be taken to ensure that the measurement volume and the sampling rate are sufficiently small/fast to resolve the microscale turbulence. Furthermore, ADVs are unable to measure flows with significant amounts of entrained air (e.g., in the surf zone with breaking waves), as the air bubbles entrained in the water column will impact the propagation of sound waves and result in unacceptable levels of error in the velocity measurements (Thorne and Hanes 2002). Finally, it is critical to keep in mind that these instruments do not account for individual suspended particles; ADVs effectively return the average velocity of all the particles in the measurement volume. Consequently, they do not return Lagrangian velocity measurements or the position of the particles within the measurement volume. 


\subsubsection{Lagrangian particle velocities}

Acoustic Doppler velocimetry has also been used in laboratory experiments to measure the velocity of discrete particles suspended in a turbulent flow. Once again making the assumption that these particles faithfully follow the fluid motion, Mordant et al. (2001, 2004, 2005) used acoustic Doppler velocimetry to measure the $3 \mathrm{D}$ Lagrangian flow velocity in the region between two counter-rotating disks (von Karman swirling flow), and Gervais et al. (2007) used it to measure the 3D Lagrangian flow velocity in a turbulent air jet. In contrast, Qureshi et al. $(2007,2008)$ used acoustic Doppler velocimetry to measure the one-dimensional velocity of inertial particles (particles that cannot be assumed to follow the fluid motion) affected by grid-generated turbulent flow in a wind tunnel to compare the particle motion to the fluid motion.

In these experiments, the velocity of each particle is determined individually (Lagrangian velocity), and as a result it is possible to compute Lagrangian turbulence statistics from the data (Gervais et al. 2007). Understanding the Lagrangian characteristics is particularly important in flows where turbulent mixing and mixing efficiency are critical, such as rain formation in clouds (Shaw and Oncley 2001; Falkovich et al. 2002) or combustion dynamics (Mordant et al. 2005). However, the particle seeding density for Lagrangian measurements must be vastly lower than that required for accurate measurements with commercially available $\mathrm{ADVs}$ so that the individual particle can be distinguished. For example, Mordant et al. (2002) only report tests with a single particle in the measurement volume-though this extreme case is also due to the use of continuous insonification, rather than the pulsed insonification (Takeda 1986) used in commercially available anemometers.

\subsection{Particle tracking with acoustics}

\subsubsection{Tracking single particles with transducers}

The Lagrangian acoustic measurement technique described in Mordant et al. $(2004,2005)$ is not only able to measure the $3 \mathrm{D}$ velocity of individual tracer particles but also tracks the particle position within the measurement volume. Consequently, it merits additional discussion. Recall that Mordant et al. (2004, 2005) used a single emitting transducer and two orthogonal receiving transducer arrays to determine the $3 \mathrm{D}$ velocity of each particle in the measurement volume (Figure 2-1). Each 
transducer array contains nine receiving transducers arrayed in the shape of a cross, such that the array forms two orthogonal linear antennas. The linear antennas are used to determine the direction of the incoming sound waves and when used in concert, the particle position.

Consider the case of an acoustically distant source of sound (the sound scattered by the suspended particle) propagating in a direction that forms an angle $\theta$ relative to the axis of one linear antenna (Mordant et al. 2005). The relative phase shift between the sound recorded by two consecutive elements of the linear array will have the phase shift, $\phi$ (Mordant et al. 2005):

$$
\phi=\frac{\omega s}{c} \cos \theta
$$

where $\omega$ is the angular frequency of the incoming sound wave, $s$ is the distance between the transducers in the linear array, and as before, $c$ is the speed of sound in the fluid. This phase shift can be related to a spatial frequency allowing the incident angle $\theta$ to be solved for using the same approximate maximum likelihood algorithm used to extract the Doppler shift in frequency from the signal (Mordant et al. 2002). Using this procedure, each of the linear antennas yields an angle of the direction of the incoming scattered sound, and the two orthogonal antennas in each transducer array yield the line along which the scattered sound propagates. The two orthogonal transducer arrays yield two lines along which the scattered sound propagates to each transducer array, and the particle position is the point where the two lines intersect (Mordant et al. 2005) (Figure 2-1).

The acoustic particle tracking system described in Mordant et al. (2004, 2005) is able to track small particles $(0.25 \mathrm{~mm})$, and has impressive temporal resolution $(0.5 \mathrm{~ms})$, yet does have several important limitations. The measurement volume is necessarily very small (e.g., $\sim 6 \mathrm{~cm} \times 6 \mathrm{~cm} \times$ $3 \mathrm{~cm}$ in Mordant et al. [2005])-the intersection of the emitting and two receiving transducer volumes. Furthermore, it is only capable of tracking one particle or at most, a few particles (Mordant et al. 2005). This is acceptable for the intended application (quantifying the Lagrangian velocity of suspended particles) but obviously does not allow for quantifying Eulerian velocity fields and spatial gradients of velocity. 


\subsubsection{Particle tracking with an acoustic camera}

One study (Wu et al. 2008) examines the possibility of using acoustic cameras to track particles. Acoustic cameras, or imaging sonars, are small multi-beam active sonars used to "see" in low-visibility environments. They transmit sound pulses into the measurement volume and use the returning sound to reconstruct an image. The range to the scattering object is determined by the travel time of the sound wave (Urick 1983). The direction to the scattering object relative to the camera is determined by "beamforming" (or "spatial filtering")-using the observed lag between adjacent transducers observing a sound returning to the transducer array to estimate the direction (Van Veen and Buckley 1988). The linear antenna used to estimate the direction to the sound source described in the Mordant et al. (2005) acoustic particle tracking system is an example of beamforming.

The frequency of the sound pulses transmitted from the acoustic camera partially dictates the operating range and resolution of the reconstructed image. In general, lower-frequency (longer wavelength) pulses can penetrate farther into the water column relative to higher-frequency pulses (shorter wavelength), at the expense of spatial resolution. The physical explanation for this phenomenon concerns the relationship between the wavelength of the sound pulse relative to scale of the spatial features of the object and the effect this has on the intensity of the scattered sound. This will be discussed in more detail in Section 3.2.3.

Wu et al. (2008) used a DIDSON dual-frequency identification sonar acoustic camera (DIDSON, Sound Metrics Corp., Bellevue, Washington) to track five $8 \mathrm{~mm}$ plastic beads. These beads were located at the water surface and were mechanically driven by a variable speed motor rather than suspended in the flow. The beads were also observed with a chargecoupled device (CCD) camera to compare the results of the acoustic camera particle tracking with the optical-camera particle-tracking results (see Figure 2-5 for Wu et al. [2008] experiment configuration). Once the physical particle position is determined from the images using geometric calibration, the particle trajectories can be differentiated using central differencing to determine the particle velocity. Wu et al. (2008) showed that the acoustic camera was able to track the particles, but the acoustic images were less smooth than the CCD camera images, leading to noisier data and higher estimates of the fluctuating velocities of the particles. On average, the mean velocity of the beads as estimated by the acoustic 
camera was only 3.8\% larger than the mean velocity estimated by the optical camera. However, the mean of the velocity fluctuations as estimated by the acoustic camera was on average $170 \%$ larger than the mean of the velocity fluctuations as estimated by the optical camera.

Wu et al. (2008) hypothesized that this large difference in the mean of the velocity fluctuations was due to the difference in spatial resolution between the acoustic and optical cameras. Therefore, they elected to also compare the acoustic camera results to the results from the optical camera with the spatial resolution of the optical camera images downgraded to match the average spatial resolution of the acoustic camera. On average, the mean of the velocity fluctuations estimated by the acoustic camera was only $2.1 \%$ larger than the mean of the velocity fluctuations estimated by the optical camera with downgraded spatial resolution, indicating that the two techniques yield comparable results once the difference in spatial resolution between the acoustic and the optical camera is accounted for.

Figure 2-5. Schematic detailing the setup of the Wu et al. (2008) experiment. Figure reproduced from Wu et al. (2008) with the permission of AIP Publishing.

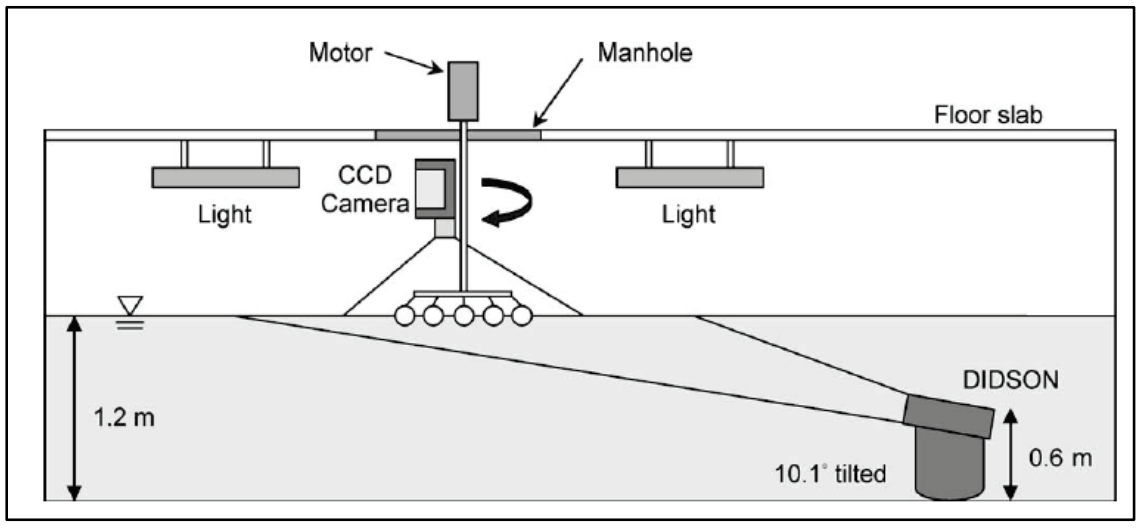




\section{The Possibility of Particle Tracking Velocimetry (PTV) and Particle Image Velocimetry (PIV) Using Acoustic Cameras}

In the previous section, there was a description of the work of Wu et al. (2008) investigating the possibility of using acoustic cameras to conduct particle tracking velocimetry. However, the limitations of that study were not discussed, and those limitations merit particular scrutiny. Wu et al. (2008) tracked comparatively large diameter beads ( $8 \mathrm{~mm}$ )-recall the acoustic systems described in Mordant et al. (2005) used much smaller diameter particles $(0.25 \mathrm{~mm})$, which would still be considered on the large side of the particles used to measure flow velocities in traditional particle tracking velocimetry with optical cameras. Additionally, these particles were located in a fixed plane by the mechanical device used to drive them, and that fixed plane was known a priori. Finally, the mechanically driven particles are not suspended in the flow or affected by the fluid motion and therefore are clearly not "tracer" particles assumed to follow the fluid motion. Consequently, the experiment described in Wu et al. (2008) is not acoustic-camera-based particle tracking velocimetry in the same sense as particle tracking velocimetry used for flow measurement.

There are several key deficiencies in the existing knowledge regarding the use of acoustics to track particles. Sophisticated 3D acoustic particle tracking systems (e.g., Mordant et al. 2005) are only able to track a single particle (or very few particles) in a small measurement volume and thus cannot be applied to larger-scale systems or used to estimate Eulerian velocity fields and spatial gradients of velocity. Acoustic cameras may offer the capability to track a large number of particles in a plane as is required in traditional optical PIV and PTV flow measurement. However, as described above, the only study (Wu et al. 2008) investigating the use of acoustic cameras for particle tracking did not close the gap between generic particle tracking and particle tracking required for flow measurement.

Several concerns will have to be addressed before acoustic-camera images can be used in traditional particle tracking techniques to measure fluid motion (PTV and PIV). This section will provide some foundational information on those techniques and then describe the aforementioned concerns that need to be considered before acoustic camera images can be 
used to make those measurements. Note that the Wu et al. (2008) study is the only one the authors of this report located during a thorough literature review that considers the feasibility of acoustic cameras for any particle tracking application. The authors of this report found no study that directly implements PTV or PIV to measure fluid motion using images from acoustic cameras. Consequently, any discussions of ways in which images from acoustic cameras may be implemented in PTV and PIV are speculative and must be verified experimentally.

\subsection{Brief overview of optical PTV and PIV}

Traditional PTV and PIV are experimental techniques to acquire instantaneous velocity measurements in fluid flows. The fluid of interest is seeded with tracer particles that reflect light and are assumed to faithfully follow the motion of the fluid-a valid assumption so long as the particles are sufficiently small (Adrian and Westerweel 2011). The region of interest in the particle-laden fluid is illuminated with a light sheet (generally from a laser) that reflects off the tracer particles. The motion of the lightreflecting tracer particles is observed, generally with a CCD or complementary metal-oxide semiconductor (CMOS) digital camera for modern PTV and PIV systems, and used to estimate the flow velocity. Figure 3-1 shows an example schematic of a traditional PIV experimental setup.

Figure 3-1. Schematic detailing a traditional optical PIV experimental setup.

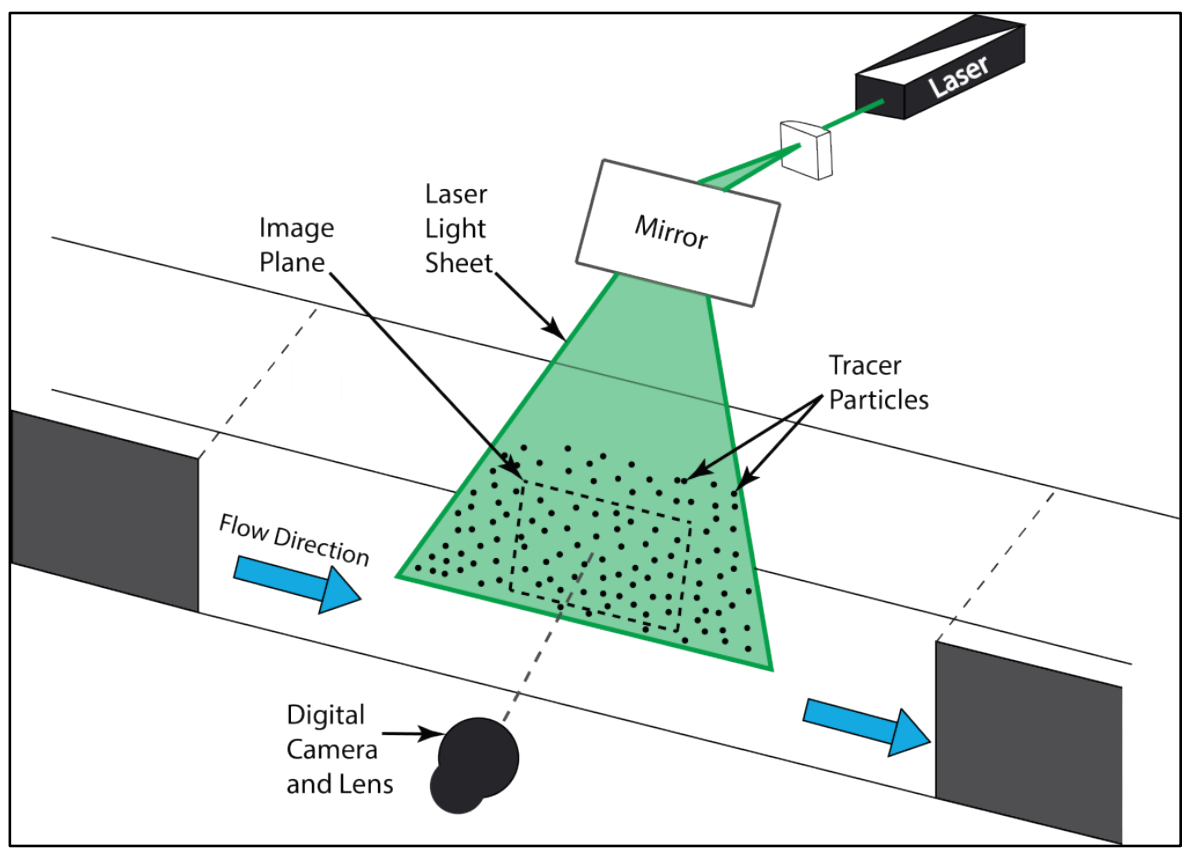


PTV is a Lagrangian technique in which the seeding density of the particles is sufficiently low such that individual particles can be tracked between frames, generating a constellation of irregularly spaced velocity vectors that are essentially the displacement of each individual particle divided by the time between images (Maas et al. 1993; Malik et al. 1993). In PIV systems, the seeding density is substantially greater, such that individual particles cannot be tracked from one frame to the next. Rather, the camera images are sub-divided into windows (e.g., $16 \times 16$ pixels), and crosscorrelation is used to determine the aggregate displacement of all the particles in each window between images (Raffel et al. 2007), making PIV effectively an Eulerian measurement technique. Figure 3-2 shows the relative particle seeding densities required for PTV and PIV analysis.

Figure 3-2. (a) Relative seeding density to track aggregate motion of particles in a sub-window (PIV). (b) Relative particle seeding density required to track individual particles (PTV). Note that the seeding density is far less for PTV, such that individual particles can be tracked between subsequent images.

(a)

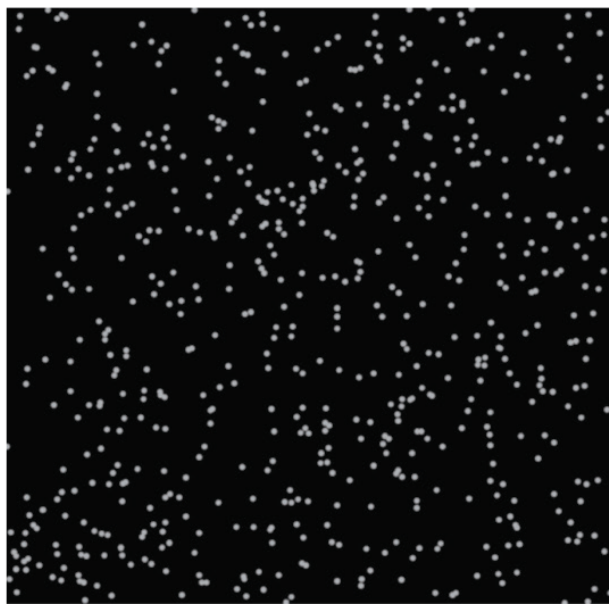

(b)

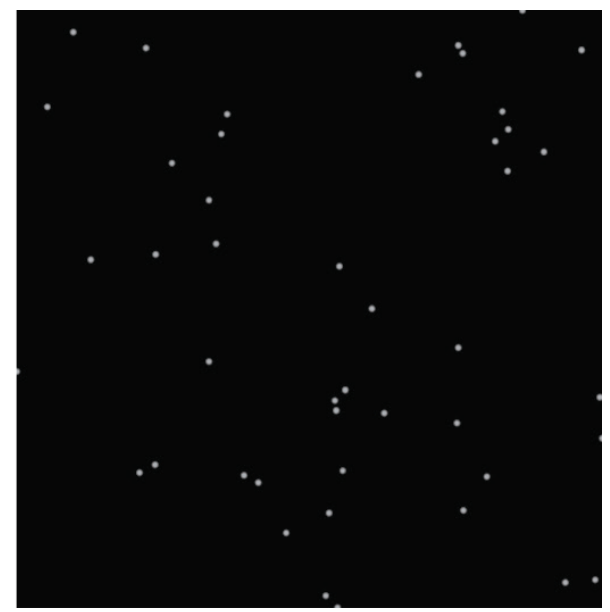

The key advantage of optical PIV over other flow measurements systems, such as hot-wire anemometry, laser Doppler velocimetry, or acoustic Doppler velocimetry, is that PIV produces $2 \mathrm{D}$ velocity vector fields rather than a single velocity point measurement (Raffel et al. 2007). Some of the more advanced PIV systems are able to capture $3 \mathrm{D}$ velocity vectors in the plane of the laser sheet (Stereoscopic-PIV-Arroyo and Greated 1991; Prasad and Adrian 1993; Soloff et al. 1997; Westerweel and van Oord 2000; Hill et al. 2000; Prasad 2000; van Doorne and Westerweel 2007) or even a fully 3D velocity vector field (e.g., Tomographic PIV-Elsinga et al. 2005, 2006a, 2006b; Weineke 2008; Scarano 2013). The ability to obtain $2 \mathrm{D}$ and $3 \mathrm{D}$ velocity vector fields allows researchers to quantify spatial gradients in velocity and consequently, components of the strain rate and 
vorticity. Optical PTV also produces $2 \mathrm{D}$ velocity vector fields, but the vectors are located wherever the particles are in the flow and thus are irregularly spaced (Maas et al. 1993; Malik et al. 1993). Consequently, to obtain spatial velocity gradients from PTV measurements, the velocity vectors must be binned and spatially averaged during post processing. Furthermore, PTV and PIV are non-intrusive flow measurement techniques that do not disturb the flow. With the ever-improving capabilities of digital cameras and increasing availability and performance of high-speed computational power, modern researchers are far less troubled by the temporal resolution concerns that plagued early pioneers of optical PTV and PIV (Adrian 1986, 1991; Westerweel 1993; Maas et al. 1993).

These attributes make PIV and PTV popular measurement techniques in laboratory experimental fluid mechanics across a broad range of research areas, such as (1) measuring flows in turbulent boundary layers (Atkinson et al. 2009); (2) measuring turbulent pipe flow (van Doorne and Westerweel 2007); (3) quantifying flow around zooplankton (Murphy et al. 2012; Adhikari et al. 2015); (4) examining zooplankton-turbulent flow interaction (Young 2014; Webster and Young 2015; Webster et al. 2015); and (5) quantifying the three-phase flow of landslide-generated tsunamis (Fritz et al. 2003a; Fritz et al. 2003b; Mohammed et al. 2011; McFall 2014).

There are comparatively few studies that employ standard planar PIV measurement techniques with laser-sheet illumination in the field. Those that attempt to do so require specially designed apparatuses (e.g., Doron et al. 2001; Smith et al. 2002; Katija et al. 2011; Smith and Friedrichs 2015) primarily because optical PTV/PIV is equipment intensive, requires stationary well-calibrated images, and can only be performed in a reasonably transparent fluid (such that the optical camera can see the light reflected by the particles). Obviously these requirements quickly become restrictive in field deployments. PIV measurement techniques can be applied to obtain surface velocity estimates from naturally illuminated images in field applications (e.g., estimating the surface velocities of tsunamis using survivor videos [Fritz et al. 2006, 2012]). This approach does not require a laser to illuminate the measurement plane, significantly reducing the equipment requirements. However, only surface velocities can be estimated in this manner, and the accuracy of the estimates is 
impaired by surface motion and the effect it has on the tracer particle surrogates.

It may be possible to address the turbid water issue and partially address the extensive equipment requirements, which prevent extension of traditional PTV and PIV techniques to field studies, by acquiring the images with an acoustic camera. Acoustic cameras such as the Sound Metrics ARIS Explorer 3000 (Figure 3-3) can easily operate in turbid environments with low optical clarity (Belcher et al. 2001; Kim et al. 2005; Belcher 2006; Wu et al. 2008) as can the acoustic Doppler velocimetry techniques described previously. Furthermore, as the acoustic camera both emits acoustic waves and receives them, it performs the primary functions of both the laser and the camera in a traditional PTV/PIV system, significantly mitigating the size and power requirements that have proven prohibitive to performing traditional PTV/PIV in the field. However, significant challenges will have to be overcome before acoustic camera images can be used for PTV or PIV. These apparent challenges will be discussed in the subsequent sections; however, it is inevitable that additional issues not discussed in this document will arise during future work investigating the use of acoustic camera images in PTV/PIV (as with any cutting-edge research).

Figure 3-3. Image of a Sound Metrics ARIS Explorer 3000 Imaging Sonar (Sound Metrics Corp., Bellevue, WA). Reprinted from www.soundmetrics.com/Products/ARIS-Sonars/ARIS-Explorer-3000 with permission.

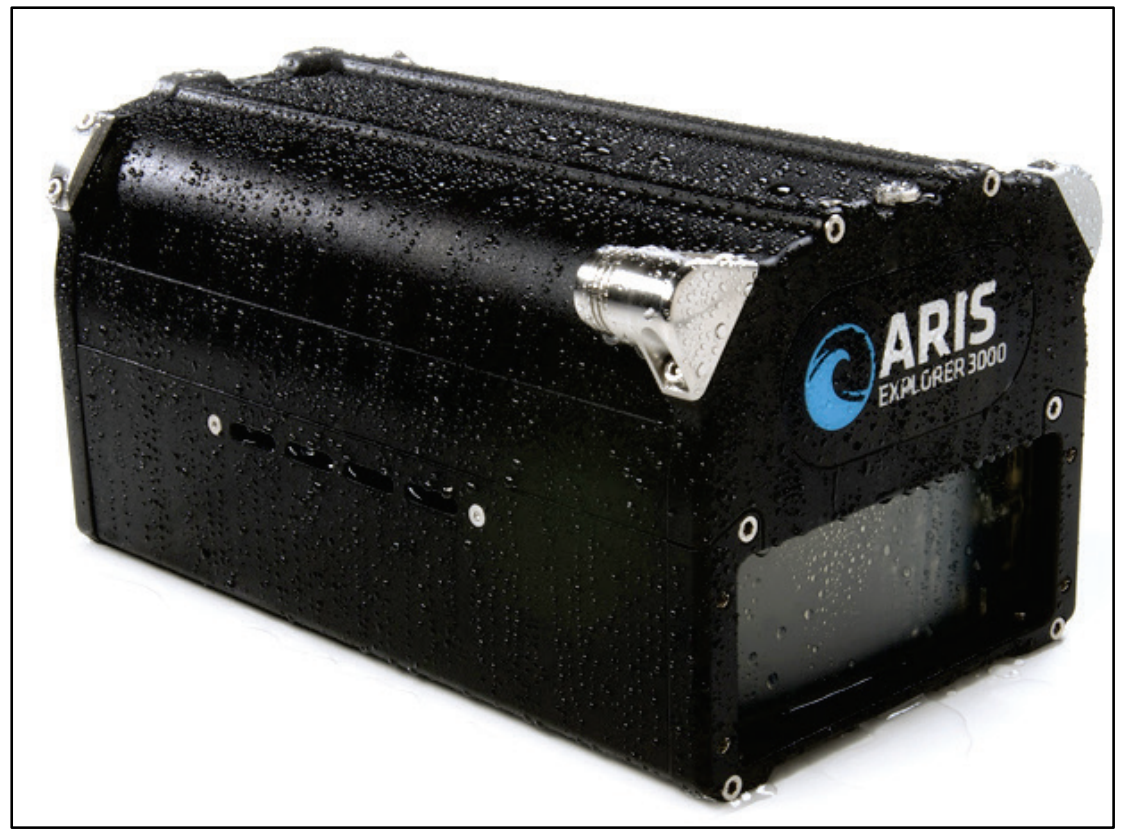




\subsection{Considerations and limitations}

Several issues need to be resolved before acoustic cameras can be successfully used in place of traditional optical cameras in standard PTV/PIV analysis. Some of these are unique to the use of acoustic images, but the majority are intrinsic to particle-tracking-based flow measurement techniques and have been wrestled with throughout the development of modern optical PTV/PIV. Therefore, in many respects this discussion is reminiscent of the earliest days of PTV and PIV and the limitations of then-current digital camera technology.

\subsubsection{Acoustic-camera resolution and frame rate}

The first consideration has to do with the resolution and frame rate of currently available acoustic cameras relative to modern digital cameras. The acoustic camera used in the 2008 acoustic camera particle tracking study of Wu et al. (DIDSON, Sound Metrics Corp.) had an initial resolution of $96 \times 512$ polar-coordinate pixels (an average of $12.8 \times 2.5$ $\mathrm{mm}^{2} /$ pixel), and the initial images were mapped to a Cartesian grid with a resolution of $505 \times 560$ pixels $\left(2.5 \times 2.1 \mathrm{~mm}^{2} /\right.$ pixel $)$ with a frame rate of 10 hertz (Hz). The CCD camera used in the same study had a frame rate of $30 \mathrm{~Hz}$ and a resolution of $720 \times 480$ pixels $\left(0.85 \times 1 \mathrm{~mm}^{2} /\right.$ pixel $)$. This frame rate and resolution is trivial for a modern research-grade CMOS digital camera and high-quality lens. For example, each Phantom v210 high-speed camera (Vision Research, Inc.) in the Tomographic PIV setup described in Murphy et al. (2012) is capable of acquiring images at 2190 $\mathrm{Hz}$, with a resolution of $1280 \times 800$ pixels $\left(0.31 \times 0.37 \mathrm{~mm}^{2} /\right.$ pixel, depending on lens configuration). Note that the DIDSON acoustic camera used in Wu et al. (2008) is not the most recently available acoustic camera, but it is unreasonable to expect improvements in the resolution and frame rate capabilities of acoustic cameras to have kept pace with the rapid advances in digital camera technology.

The resolution and frame rate limitations have important ramifications for PTV and PIV. First and foremost, the camera resolution is partially responsible for the limits on the minimum size of the tracer particles that can be observed (Raffel et al. 2007; Wu et al. 2008). The amount of scattered light-or in the case of an acoustic camera, scattered sound-that returns to the camera is also responsible for the limits on the minimum size of the tracer particles (Adrian and Westerweel 2011). In general, smaller particles more faithfully follow the fluid motion, and the 
assumption that particles faithfully follow the fluid motion is the foundation upon which all particle-tracking-based flow measurement techniques are built (Raffel et al. 2007). This will be discussed in more detail in the following section.

Second, the camera resolution limits the accuracy and spatial resolution of the resulting velocity vector fields. The spatial resolution and accuracy of the velocity vector fields essentially sets a lower bound on the spatial scale of the flows able to be measured by the technique (along with the tracer particle size). In the case of PTV for example, the estimated velocity vector for a given particle is essentially the net displacement of the particle divided by the time between frames (Maas et al. 1993; Malik et al. 1993). Errors in the particle's position in either frame will result in errors in the estimated velocity vectors. In PIV, the frames are divided into subwindows (e.g., $16 \times 16$ pixels) which need to be large enough to contain enough particles to obtain the net displacement statistically using crosscorrelation (Raffel et al. 2007). The ratio of the total number of pixels to the size of each sub-window determines the number of velocity vectors that will be in each velocity vector field, and the size of the sub-window determines the spatial resolution. The error in the estimated velocity due to the camera resolution is likely to be less in PIV than in PTV because they will effectively be spatially averaged, but may still be a concern.

Finally, the camera frame rate limits the temporal resolution of the velocity vector fields and in the case of highly unsteady flows, the accuracy of the velocity vectors themselves (Raffel et al. 2007; Wu et al. 2008). The velocity vectors yielded by both PTV and PIV are effectively estimates of the average velocity of the particle(s) over the time between frames. The shorter the time between frames, the closer these estimates become to instantaneous velocities. In steady flows this is less of a concern, but measuring highly unsteady flows with a low frame rate camera will result in velocity vector fields that are too temporally averaged to represent the instantaneous flow features.

One of the principal reasons spatial and temporal resolution are of such concern to the wider experimental flow measurement field is that many flows in engineering and nature are turbulent. Turbulent flows are highly unsteady and exhibit spatial variability at a wide range of spatial scales (Tennekes and Lumley 1972; Pope 2000). Consequently, it is desirable to have a flow measurement technique with finer spatial and temporal 
resolution than the smallest length and time scales of the fluid motion in a turbulent flow-the Kolmogorov microscales (Kolmogorov 1941).

\subsubsection{Particle dynamics and ramifications for PTV/PIV}

Unsurprisingly, understanding the motion of particles suspended in a fluid flow is of critical concern to any PTV/PIV measurement system, optical or otherwise. As previously discussed, particle-tracking-based flow measurement techniques such as PTV and PIV do not directly measure the flow velocity (Adrian and Westerweel 2011). Rather, they observe the motion of particles suspended in the flow and infer the fluid velocity based on the assumption that those particles are following the fluid motion. Mentioned previously, it is preferable to conduct PTV and PIV measurements with particles as small as reasonably possible because generally smaller particles more faithfully follow the fluid motion. This follows from the laws governing the drag on particles at low Reynolds number (Stokes' drag law). A more general discussion of particle dynamics and the relationship between suspended particles and the fluid itself is far beyond the scope of this report (Chao 1964; Hjemfelt and Mockros 1966; Maxey and Riley 1983; Mei 1996; Melling 1997; Crowe et al. 1998; Ferrante and Elghobashi 2003; Yang and Shy 2005; Lucci et al. 2011; Herrmann et al. 2012; Farazmand and Haller 2015), but some knowledge of Stokes' drag law is useful to grasp the ramifications of increasing the particle size.

Stokes' drag law is named after George Gabriel Stokes and arises out of the solution to the Navier-Stokes equations for creeping flow, sometimes referred to as "Stokes flow," around a sphere. Creeping flow is a specific type of fluid flow in which the viscous forces dominate (Kundu and Cohen 2004) and only occurs in very low Reynolds number flows $\left(R e=\frac{U d}{v} \ll 1\right.$, where $U$ is the free-stream velocity, $d$ is the sphere diameter, and $v$ is the kinematic viscosity of the fluid). Assuming creeping flow, the drag on a sphere in a uniform flow field will be inversely proportional to the Reynolds number (Stokes' drag law). As a result, the Navier-Stokes equations can be analytically solved to determine the acceleration of a sphere in a uniform flow field. Given this acceleration, it is possible to derive an expression for the difference between the velocity of the sphere $\left(V_{p}\right)$ and the free-stream velocity $(U)$, referred to as the slip velocity (Tropea et al. 2007): 


$$
V_{p}-U=d^{2} \frac{\left(\rho_{s}-\rho\right)}{18 \mu} \frac{d V_{p}}{d t}
$$

where $\rho_{s}$ is the density of the sphere, $\rho$ is the density of the fluid, $\mu$ is the dynamic viscosity of the fluid, and $\frac{d V_{p}}{d t}$ is the sphere acceleration. If the density of the sphere is much larger than the density of the fluid, the rate at which the velocity difference approaches zero can be modeled as exponential decay, in which case the velocity of the sphere as a function of time is given by (Raffel et al. 2007):

$$
V_{p}=U\left[1-\exp \left(-\frac{t}{\tau_{p}}\right)\right]
$$

The variable $\tau_{p}$ denotes the characteristic response time of the sphere and under these assumptions is equivalent to the particle acceleration coefficient in Equation (3) (Tropea et al. 2007):

$$
\tau_{p}=d^{2} \frac{\left(\rho_{s}-\rho\right)}{18 \mu}
$$

The analysis just discussed in deriving the characteristic response time of a sphere is obviously very simplified (Raffel et al. 2007). If (1) the freestream velocity changes rapidly with time, (2) the velocity or diameter are too high for Stokes' drag law to apply, or (3) the density difference between the sphere and the fluid is too small for an exponential decay law to govern the change in the slip velocity, then the equations governing particle motion become more complex (e.g., the Basset-Boussinesq-Oseen Equation [Crowe et al. 1998] or the Maxey-Riley Equation [Maxey and Riley 1983]). Nonetheless, $\tau_{p}$ is often used as an estimate of the characteristic response time of spherical tracer particles suspended in a fluid flow.

To determine how well the tracer particles will be able to follow the fluid motion, it is necessary to compare the response time of the tracer particles to the smallest time scale of the fluid flow (Tropea et al. 2007). In a turbulent flow, the smallest time scale is the Kolmogorov time scale, $\tau_{K}$ (Kolmogorov 1941), which can be estimated to an order of magnitude by

$$
\tau_{K}=\left(\frac{v L}{U^{3}}\right)^{1 / 2}
$$


where $L$ is the characteristic length scale of the flow (e.g., the water depth). If the flow is laminar, or the fine-scale turbulence characteristics are not of interest, then the time scale of the flow is often simply the ratio of the characteristic length scale to the free-stream velocity. In either case, the ratio of the response time of the particle $\left(\tau_{p}\right)$ to the characteristic time scale of the flow $\left(\tau_{f}\right)$ is referred to as the Stokes number (Stk), a dimensionless number that indicates how well the suspended particles follow the fluid motion (Lucci et al. 2011):

$$
S t k=\frac{\tau_{p}}{\tau_{f}}
$$

The Stokes number is often used as a quick estimate of how well tracer particles will follow the motion of a given flow, particularly for gaseswhere very often the tracer particles are several orders of magnitude more dense than the fluid. As a general rule, if Stk 》1, then the tracer particles will not follow the fluid motion, particularly in areas of rapid change of direction or deceleration. If $S t k \ll 1$, then the particles will closely follow the fluid motion (Tropea et al. 2007).

Equations (3), (5), and (7) make the desirable characteristics of tracer particles very clear. The particles need to match the density of the fluid as closely as reasonably possible and need to be as small as reasonably possible to minimize the characteristic response time of the particles. When conducting PTV/PIV in liquid flows, it is fairly trivial to find particles that closely match the density of the fluid-as a result, it is possible to use much larger particles in PTV/PIV experiments in liquids (Melling 1997). Nevertheless, researchers should use caution before matching the densities of the particles and the fluid, or manipulating the fluid viscosity to obtain a smaller Stokes number, and subsequently assuming the particles will adhere to the fluid motion regardless of size (Lucci et al. 2011).

If the particles are too large, than the very first underlying assumption of Stokes flow is no longer valid, specifically, that the flow must have a very low Reynolds number $\left(R e=\frac{U d}{v} \ll 1\right)$. As a consequence, the other terms in the equations governing particle motion (e.g., the Maxey-Riley equation or the Basset-Boussinesq-Oseen equation) may no longer be neglected (Mei 1996; Adrian and Westerweel 2011; Langlois et al. 2015), such as the following: 
1. The term that accounts for the effect whereby a particle accelerating through a fluid also accelerates the fluid immediately surrounding the particle due to the no-slip condition (Auton et al. 1988). This will result in a small amount of fluid being "carried" by the particle, with an effect similar to increasing the particle's mass. This is referred to as an "added-mass" term as a result.

2. The term that accounts for a lag between the development of the boundary layer on the surface of the particle and the acceleration of the particle itself (Candelier et al. 2004; Langlois et al. 2015). As a result of this lag, momentum and vorticity diffusing away from the particle through the boundary layer "remember" the prior changes in the slip velocity. This effect results in a net force on the particle, referred to as either a "history" term or the "Boussinesq-Basset memory" term, which becomes more critical as the density of the particle becomes smaller (Langlois et al. 2015).

3. The term representing the buoyancy force on the particle due to the difference in density between the particle and the fluid (Tropea et al. 2007). For sufficiently large particles this term becomes extremely important in the context of PTV/PIV experiments, as even slightly buoyant/negatively buoyant particles will not remain in suspension for the experiment duration.

4. The term representing the lift on the particle due to particle rotation and fluid shear (Adrian and Westerweel 2011).

These additional terms will result in further discrepancies between the motion of the particle and the motion of the surrounding fluid for larger particles (Adrian and Westerweel 2011).

Embedded within all this complicated analysis is a fairly intuitive concept-the tracer particles should be much smaller than the smallest scale fluid motion of interest because fluid motions and vortices that are substantially smaller than a given object will be unable to meaningfully impact the motion of that object, much less cause it to exactly follow the fluid motion (Adrian and Westerweel 2011). This is the reason experiencing turbulence during air travel is generally mildly bumpy rather than a constant dance with death-the overwhelming majority of the turbulent vortices in the air are much smaller than the aircraft and cannot significantly impact its motion. 
However, larger diameter particles scatter more light and sound (this will be discussed in more detail in the next section) and as a result can be observed and tracked when measuring a larger area or when using lowerresolution cameras (Adrian and Westerweel 2011). Due to these factors, researchers using particle-tracking-based flow measurement techniques have always been forced to find a balance with the tracer particles used in their experiments-a balance between the particle size small enough to follow the fluid motion and that large enough to scatter sufficient light (or sound) to be observed given the resolution of the camera (Melling and Whitelaw 1973; Melling 1997). As discussed in Section 3.2.1, acoustic cameras do not possess the same resolution as modern digital cameras and generate their images from scattered sound rather than light. Therefore, particles should be carefully sized based on the resolution of the acoustic camera. The size of the selected particles will place a lower bound on the scale of the flows able to be measured and an upper bound on the spatial variability and unsteadiness of the flows able to be measured (Adrian and Westerweel 2011).

\subsubsection{Plane wave scattering from particles}

Where the previous section was devoted to explaining why the tracer particles should be a small as reasonably possible, this section explains why the tracer particles may need to be larger. The scattering of both light and sound from objects involves the dynamics of plane waves (Urick 1983; Adrian and Westerweel 2011). Both light and sound can be conceptualized as plane waves, and the underlying physics governing both types of plane waves is very similar. However, acoustic waves are simpler than electromagnetic waves and can be described with the scalar velocity potential (Weill 2014). Consequently, the wave function for acoustic waves can be separated into the product of two functions-one a function of only space, and one a function of only time-to derive the Helmholtz equation. The Helmholtz equation governs the scattering of acoustic plane waves; the solution for the scattering by an elastic sphere is given by Anderson (1950) and by a solid sphere by Faran (1951). The scattering of a plane, linearly polarized, coherent electromagnetic wave by a sphere is described by the Lorenz-Mie solution to Maxwell's equations (Born and Wolf 1980; Adrian and Westerweel 2011). The general behavior of these equations governing plane wave scattering is critical to particle-tracking-based flow measurement, though the details will be omitted from this report. 
First, the observed intensity of the scattered sound and light depends on the angle of observation relative to the incident plane wave (Partridge 1993; Adrian and Westerweel 2011). The majority of acoustic-based measurements rely on backscattered sound (sound scattered directly back to the instrument) whereas most optical-based PTV/PIV systems rely on side-scattered light (as does the acoustic-based particle tracking device described in Mordant et al. 2005, albeit with scattered sound). Generally speaking, the observed intensity of back-scattering and forward-scattering of plane waves is greater than side-scattering, and this must be taken into account for optical PIV measurements. Second, the intensity of scattered light (Adrian and Westerweel 2011) and sound (Weill 2014) from a particle decreases with distance away from the particle, $r$, as $1 / r^{2}$.

Outside of the two criterion mentioned in the previous paragraph, the intensity of light scattered by particles can be easily increased in three ways. The first (and most obvious) is to increase the intensity of the incoming light or sound (Adrian and Westerweel 2011). For light, this may be accomplished by using a more powerful laser or improving the focusing of the sheet of light. Similarly, a concentrator lens may be used on an acoustic camera to focus the acoustic energy into a narrower volume (this will be discussed in more detail in the next section).

Second, the particle material also affects how efficient the particle is at scattering light/sound. For light scattering, the relevant material property is the refractive index (Adrian and Westerweel 2011), and for acoustic scattering it is the acoustic impendence (Kinsler et al. 2000), which are properties that quantify the propagation of light/sound through a given medium. The scattering is improved when the refractive index/acoustic impendence of the particle material is substantially different from that of the fluid. For example, metal-coated spheres are popular choices in optical PIV in water because the refractive index of most metals is much higher than that of water. Similarly, air bubbles are highly visible in acoustic camera images because the acoustic impedance of air is much lower than that of water.

The third way to increase the intensity of scattered light or sound from a particle is to increase the particle diameter. The exact effect of increasing the particle diameter is complex and depends on the scale of the wavelength of the light/sound plane wave relative to the particle diameter. In this respect, the behavior of acoustic plane waves and electromagnetic 
plane waves is quite different because they have substantially different wavelengths. The wavelength $(\lambda)$ of an acoustic plane wave depends on the speed of sound $(c)$ in the fluid the wave is traveling through and the frequency, $f$ (Urick 1983):

$$
\lambda=\frac{c}{f}
$$

The wavelength for the $3 \mathrm{MHz}$ acoustic waves emitted by the ARIS Explorer 3000 shown in Figure 3-3 (Sound Metrics Corp., Bellevue, WA) propagating in water is $0.5 \mathrm{~mm}$-note that this is a short wavelength for an acoustic wave. Yet, the wavelength of visible light is 400 nanometers $(\mathrm{nm})$ to $700 \mathrm{~nm}$, approximately 10,000 times smaller.

Simple relationships between the scattering of plane waves by particles and the particle diameter can be derived in two cases. The first occurs when the particle diameter, $d$, is much smaller than the wavelength of the plane wave, $\lambda$. This regime is referred to as Rayleigh scattering, and the intensity of Rayleigh scattering waves scales as $d^{4}$ (Adrian and Westerweel 2011). The second occurs when the particle diameter, $d$, is much larger than the wavelength of the plane wave, $\lambda$. In this regime, the laws of geometric optics apply (Stanton et al. 1998; Adrian and Westerweel 2011), and the scattered wave intensity scales as $d^{2}$. The intermediate regime where the particle diameter and the plane wave wavelength are of similar scales is often referred to as Mie scattering. In this regime, the approximations that allow for the derivation of simple relationships between the scattering intensity and the particle diameter no longer apply.

From the relationships just described, one can easily explain why acoustic cameras are able to "see" in turbid water where optical cameras cannot. The wavelength of the acoustic plane waves is much longer than the finescale particles suspended in the water, placing them in the Rayleigh scattering regime (scattering intensity scales as $d^{4}$ ). Since the diameter of these particles is much less than unity, the scattering of the acoustic plane waves by them is negligible, and as a result, acoustic plane waves can penetrate much farther into the water column. Conversely, only incredibly fine suspended material ( $\sim 50 \mathrm{~nm}$ or smaller) will fall into the Rayleigh scattering regime for visible light $(\lambda=400-700 \mathrm{~nm})$. As a result, more light is scattered by these particles, inhibiting the light from penetrating deeper into the water column. 
This also explains the importance of larger particles for particle tracking systems. For traditional optical PIV with laser light, it is trivial to use small particles and still remain in the $d \gg \lambda$ regime, where the scattered wave intensity scales as $d^{2}$ and the particles easily scatter sufficient light to be detectable by an optical camera. However, the seeding particle sizes traditionally used for PIV fall into the Mie scattering regime, or even the Rayleigh scattering regime (scattering intensity scales as $d^{4}$ ), for acoustic plane waves; thus, it is expected to see a more rapid decrease in scattered sound with decreasing particle size when using an acoustic camera.

\subsubsection{Out-of-plane particles and out-of-plane fluid motion}

In this section, there is a description of two sources of error in PTV and PIV measurements due to out-of-plane effects. The first occurs due to particles that are not precisely in the measurement plane because a truly 2D measurement plane is a purely abstract concept and does not exist. As previously described, in 2D optical PTV/PIV the measurement plane is illuminated by a sheet of laser light. The sheet of light is not truly a 2D plane because it has a finite thickness and is rather a very thin column of light. A full discussion of the laws governing this sheet of light and the properties of the optics used to generate it are beyond the scope of this report (see Hecht and Zajac 2001), but some qualities of a beam of laser light are important to bear in mind.

The thickness of a beam of light is not a constant but contracts to a minimum diameter, the beam waist, then continuously expands once it has passed it (Adrian and Westerweel 2011). Furthermore, the rate at which the beam expands once it has passed the beam waist is inversely proportional to the beam waist (Durst and Stevenson 1979). As a result, thinner sheets of laser light expand more quickly and can only be maintained over shorter distances than thicker sheets (Adrian and Westerweel 2011). Finally, the light intensity is not constant across the laser sheet-either across the sheet thickness or across the plane extents. Rather, the distribution of light intensity depends on the original distribution of light intensity across the beam of laser light before it was expanded into a sheet (often Gaussian) and the optics used to expand the beam (Raffel et al. 2007). For reference, the typical desired thickness $\left(\Delta z_{0}\right)$ of a laser light sheet for optical PIV is on the order of $1 \mathrm{~mm}$ (Adrian and Westerweel 2011). 
As a consequence, it is possible to observe particles that are actually at different depths within the column of light but appear to be in the same 2D plane. This will introduce errors in the reconstructed velocity fields if significant $3 \mathrm{D}$ spatial variability exists in the flow. In optical PIV, errors due to the observation of out-of-plane particles are minor because the laser sheets are very thin (Raffel et al. 2007). Therefore, all the observed particles are necessarily very close to the same plane. However, this will not be the case with images from an acoustic camera. To explain by way of example, the ARIS Explorer 3000 shown in Figure 3-3 has a field of view of $30^{\circ} \times 14^{\circ}$. A diagram of the resulting insonified measurement volume is shown in Figure 3-4.

Figure3-4. Diagram of the plan view and the side view of the measurement volume of a Sound Metrics ARIS Explorer $\mathbf{3 0 0 0}$ Imaging Sonar.

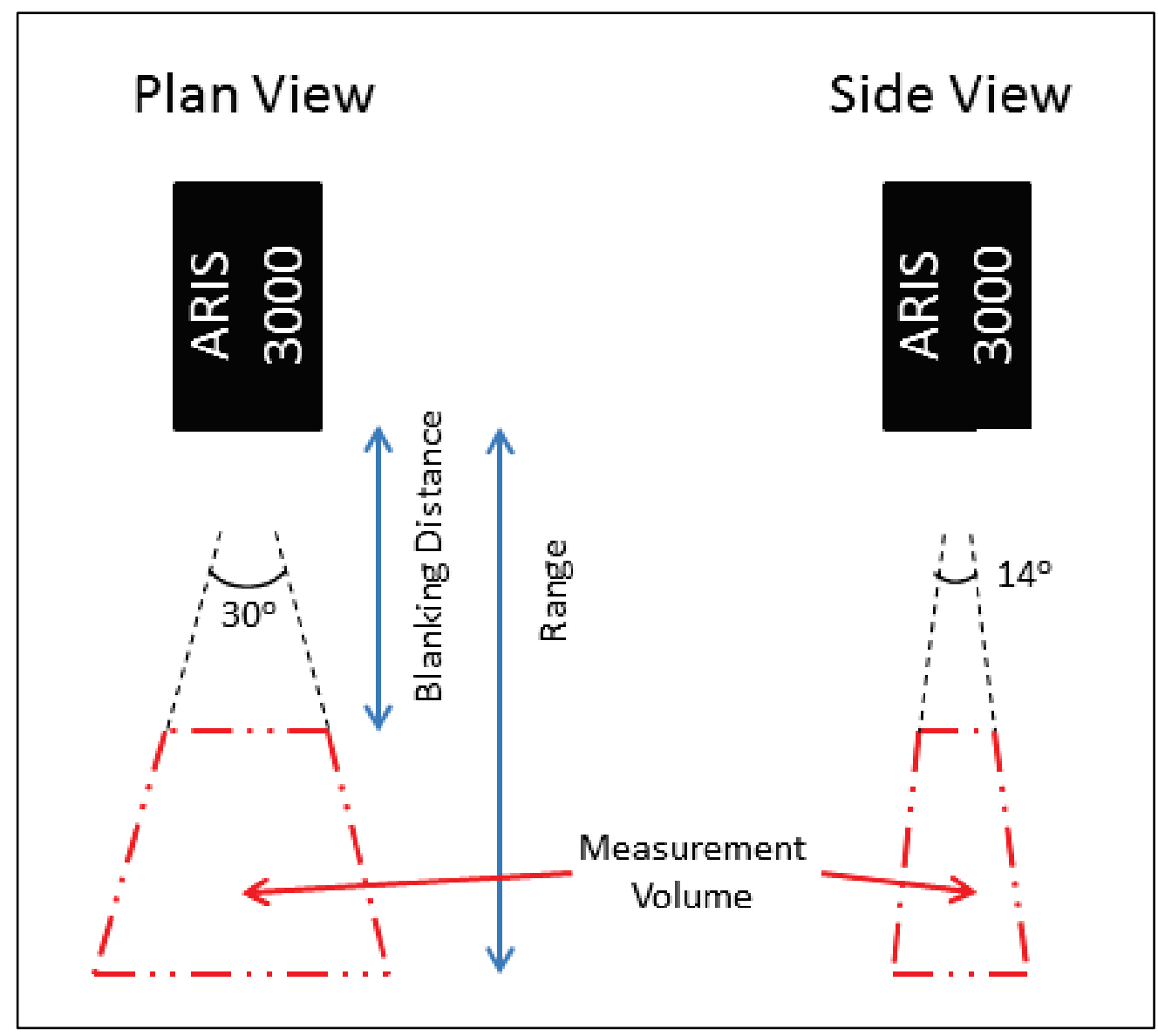

Obviously, the measurement volume in this configuration is not a reasonable facsimile of a $2 \mathrm{D}$ plane. Unfortunately, the acoustic camera is only able to distinguish the 2D position of objects in the measurement volume (i.e., its position in the plan view) and cannot determine its precise 
position in the out-of-page dimension (the left-to-right dimension in the side view). Even assuming the minimum recommended blanked distance and shortest range, the thickness of the insonified volume (the out-ofplane dimension in the top-down view) ranges from 28 centimeters $(\mathrm{cm})$ to $55 \mathrm{~cm}$. This means that particles that appear to be in the same physical position could be separated by more than half a meter, resulting in massive errors in the estimated velocity if the flow possesses variability in the out-of-plane direction. Wu et al. (2008) avoided this complication altogether in their study because the particles they tracked were all on the surface, and as a result, they knew the plane of the particle motions a priori.

Fortunately, the $14^{\circ}$ spreading angle of the acoustic camera can be reduced to $1^{\circ}, 3^{\circ}$, or $8^{\circ}$ using the appropriate concentrator lens-without altering the frequency of the camera. Again assuming the minimum recommended blanked distance and shortest range, the thickness of the insonified volume for a camera equipped with a $1^{\circ}$ concentrator lens ranges from $2 \mathrm{~cm}$ to $4 \mathrm{~cm}$. This is still substantially larger than the $1 \mathrm{~mm}$ thick laser sheets of optical PIV but should be sufficiently thin to measure flows that lack substantial variability in the out-of-plane direction. An added benefit of using a concentrator lens is that it focuses the acoustic energy into a narrower volume, increasing the resolution of the images. Note that mitigation of the errors due to the thickness of the laser sheet/insonified volume must be balanced against the need to observe a sufficient number of particles for PIV analysis-a requirement more easily met with a thicker measurement volume.

The second source of error due to out-of-plane effects is the out-of-plane motion of in-plane particles. This issue stems from the fact that a single optical camera viewing a particle experiencing out-of-plane motion will incorrectly map the out-of-plane displacement back to an apparent (but incorrect) in-plane displacement, even if the instantaneous particle position is very near the measurement plane (Prasad 2000). This type of error is referred to as "perspective error" and is described in more detail in Prasad (2000). Perspective error is present in any 2D single opticalcamera particle tracking system. Consequently, if a flow possesses significantly large velocities in the out-of-plane direction, then Stereoscopic PIV, which features two cameras viewing the same plane to quantify the out-of-plane velocity component, will yield improved estimates of the in-plane velocity components over traditional 2D optical 
PIV (Raffel et al. 2007). It is possible that a 2D single-camera particle tracking system employing an acoustic camera to obtain images may be less affected by this source of error than traditional optical-camera systems because the acoustic camera yields a more direct measurement of the physical position of the particles. 


\section{Acoustic Cameras for Bubble Image Velocimetry (BIV)}

Mentioned briefly in Section 3.2.3, bubbles are highly visible in acousticcamera images because the acoustic impedance of air is substantially lower than that of water. This raises the possibility that air bubbles themselves could potentially be used in place of tracer particles to estimate the velocities from acoustic-camera images. Air bubbles are generally to be avoided in traditional optical PIV because their presence will obstruct the visibility of the tracer particles and lead to gaps in the optical-camera images where no tracer particles exist (i.e., inside the air bubbles); no velocity vectors can be obtained in these gaps. Furthermore, the presence of the bubbles will disrupt the motion of the tracer particles and may lead to errors in the reconstructed velocity (Adrian and Westerweel 2011). Consequently, PIV with conventional tracer particles is ineffective in highvoid fraction two-phase flows, such as breaking waves or elsewhere in the surf zone (Ryu et al. 2005).

However, there exists a particular subclass of PIV that uses the air bubbles themselves as tracer particles. Several different configurations have been devised to accomplish this with optical cameras. Govender et al. (2002a, b) simply substituted air bubbles for tracer particles and otherwise used a traditional PIV setup with a sheet laser light and an optical camera to track the bubble motion. Others have done away with the laser sheet and rather placed a light source immediately opposite the optical camera to backlight the air bubbles, relying on a narrow depth of field in the camera images to ensure the bubbles are close to the measurement plane. This technique is referred to as "shadowgraphy" because the bubbles appear as shadows in the camera images (Hassan et al. 1998; Nishino et al. 2000; Lindkin and Merzkirch 2001). Building upon the shadowgraphy technique, more recent efforts have used back- and front-lighting in an attempt to better distinguish individual bubbles (Ryu et al. 2005, 2007; Ryu and Chang 2008; Chang et al. 2011; Pedrozo-Acuña et al. 2011; Lin et al. 2012).

\subsection{Bubble image velocimetry}

The technique proposed by Ryu et al. (2005) is the one generally referred to in the literature as "bubble image velocimetry" or BIV. As described above, in this method bubbles are used in place of tracer particles, and the measurement plane is illuminated from a light source both directly 
opposite the optical camera and a light source located above the camera facing the measurement plane at an angle $60^{\circ}$ down relative to the camera line-of-sight. The bubbles appear as shadows in the camera images (as in the "shadowgraphy" technique described above), and the image intensities are inverted such that the now high-intensity regions correspond to the bubbles. The camera depth of field is used to constrain the observed bubbles to those close to the measurement plane (i.e., at the focal point of the camera). The images are processed using traditional PIV software (such as DaVis from LaVision, Inc. in the case of Ryu et al. [2005]). Ryu et al. (2005) performed a simple validation experiment to quantify the rise velocity of bubbles in a bubble plume using both the proposed BIV technique and fiber optic reflectometry (FOR) (Chang et al. 2003) to assess the accuracy of the BIV-determined bubble velocities. The mean velocities of the BIV estimates were within $1 \%$ of the FOR estimates, although there were noticeable differences in the instantaneous velocities.

The advantage of BIV over the shadowgraphy technique is that a wider range of bubble sizes and densities of bubbles (void fractions) are quantifiable because individual bubbles are more easily distinguishable in the camera images. Relative to the Ryu et al. (2005) BIV technique, shadowgraphy requires a lower void fraction and smaller bubbles to be effective, which limits its applicability in measuring extremely high void fraction two-phase flows like breaking waves (Ryu et al. 2005). BIV and other bubble-tracking-based velocimetry techniques have been used to quantify the velocity in high void fraction two-phase flows where traditional PIV is ineffective, such as (1) in the surf zone or other areas with breaking waves and significant air entrainment (Govender et al. 2002a, b; Pedrozo-Acuña et al. 2011); (2) the motion of water from plunging breaking waves overtopping structures, referred to as "green water" (Ryu et al. 2005, 2007; Ryu and Chang 2008; Chang et al. 2011) (Figures 4-1, 4-2); and (3) the velocities and flow structure inside a hydraulic jump (Lin et al. 2012). 
Figure 4-1. Sample image of a plunging breaking wave impacting on the model structure in Ryu et al. (2005). The region boxed in green delineates a highly aerated region in which BIV must be used to obtain velocity measurements. Figure reprinted from Ryu et al. (2005); IOP

Publishing. Reproduced with permission. All rights reserved.

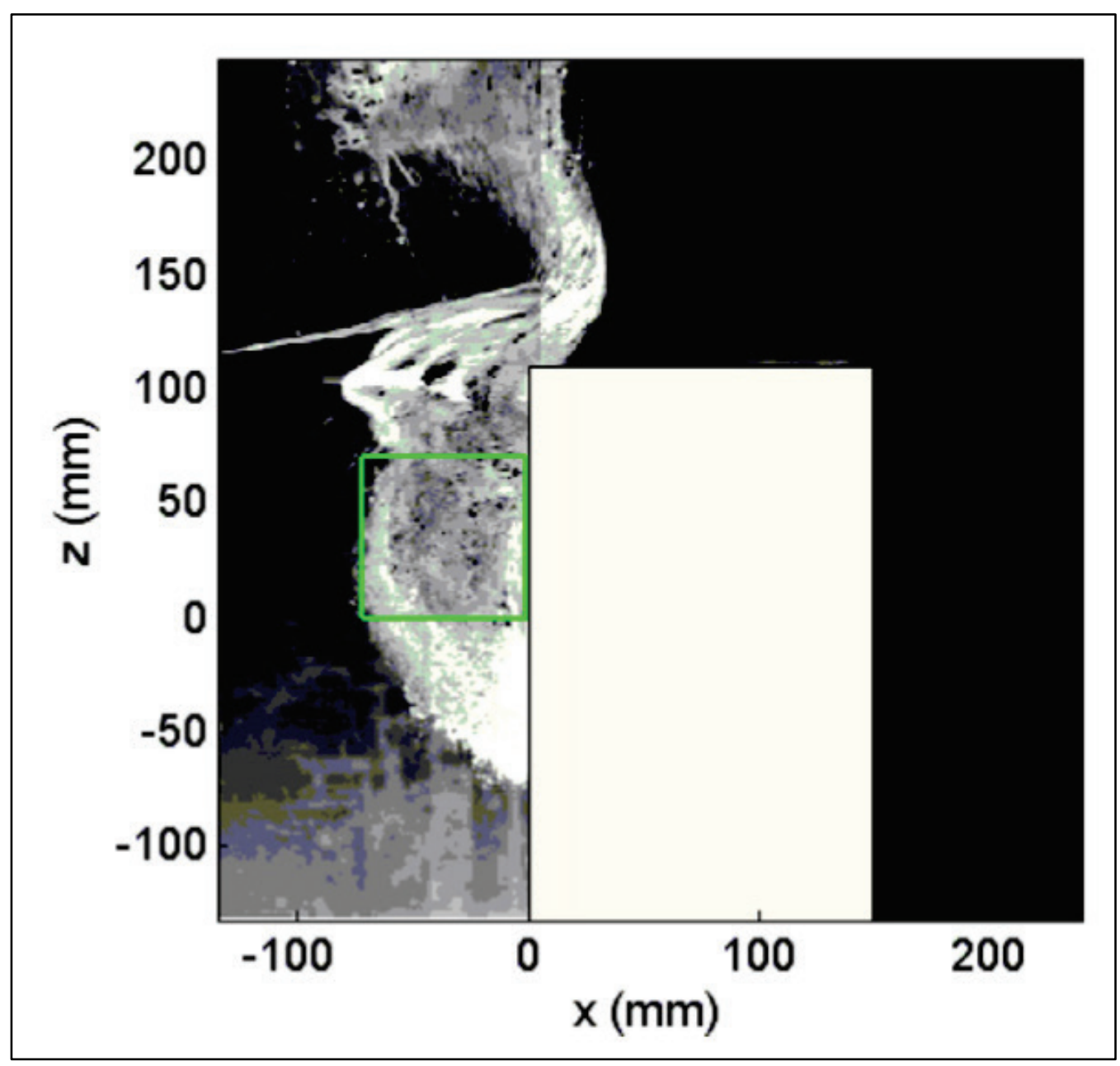


Figure 4-2. Mean velocity field of the plunging breaking wave impinging on the model structure measured with BIV (Ryu et al. 2005). Note the measurement area is confined to the high void fraction region of the flow. Figure reprinted from Ryu et al. (2005); IOP Publishing. Reproduced with permission. All rights reserved.

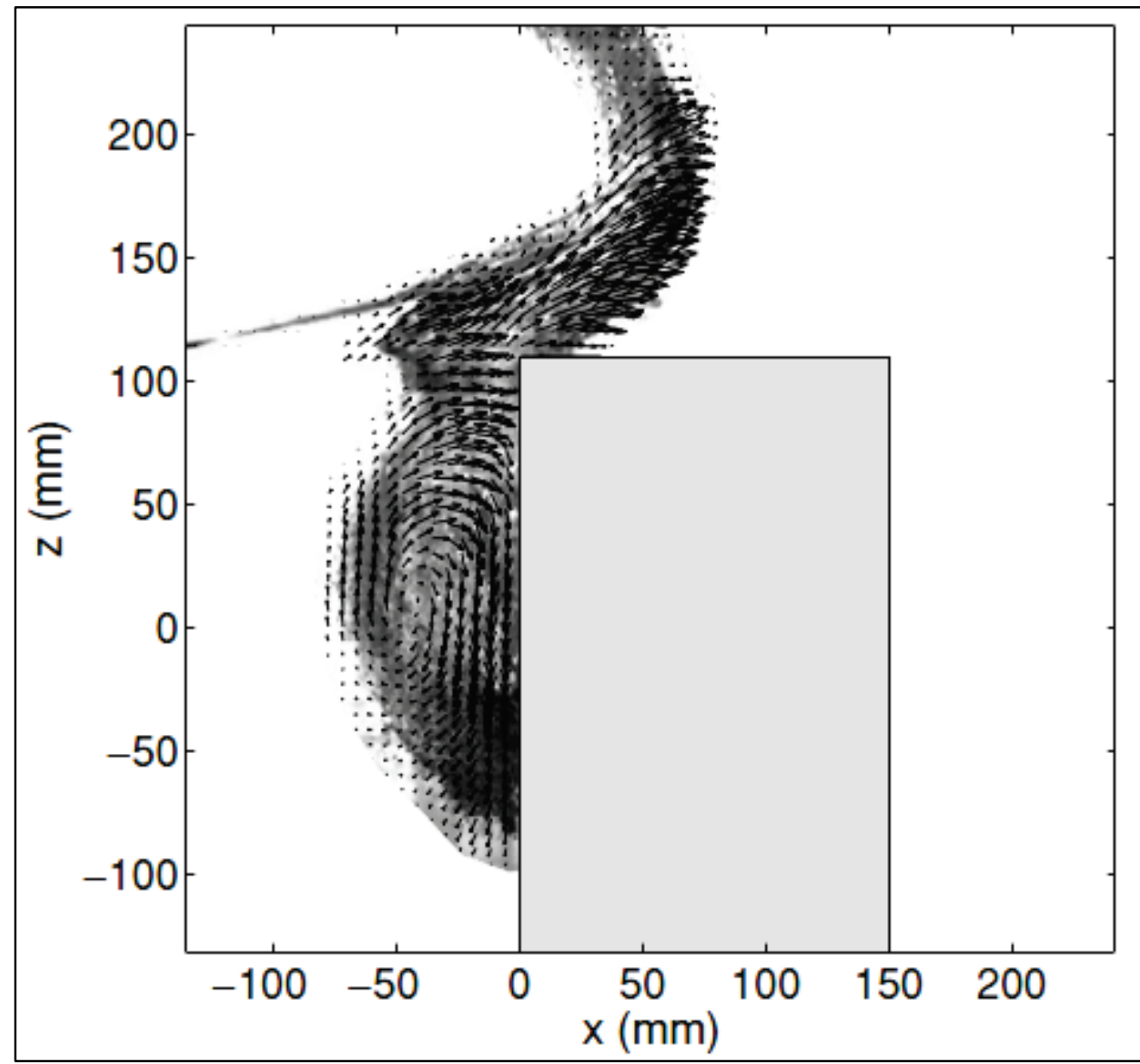

It is important to emphasize that these flows (in particular breaking waves) have proven incredibly challenging to quantify both experimentally and numerically. Not only are they complex, highly turbulent flows, but the amount of entrained air makes measurements of these flows essentially unobtainable with other more common measurement techniques and devices (ADCP, ADV, traditional PIV). Consequently, comparatively little is known about their velocity structure, and it is critical to keep this in mind when discussing the limitations of the BIV measurement technique.

\subsection{Bubble size considerations}

BIV is an intriguing new technique due its potential to quantify previously intractable flows, but significant concerns will have to be addressed before 
it will gain the widespread acceptance of traditional PIV. These concerns stem from the effectiveness of bubbles as tracer particles that faithfully follow the flow. The properties of flow-following tracer particles are well described in Section 3.2.2; in brief, it is desirable to have tracer particles that are as small as possible and as close to the density of the fluid as possible. Although air is approximately one thousand times less dense than water, this does not necessarily preclude the use of air bubbles as tracer particles. Indeed, liquid droplets are often used in PIV experiments of gas flows, despite the large density difference (Melling 1997; Raffel et al. 2007; Adrian and Westerweel 2011). However, air bubbles must be much smaller than density-matched tracer particles in order to faithfully follow the motion of liquid water (this follows from Equations (3), (4), and (5) in Section 3.2.2.).

Furthermore, the buoyancy force on small air bubbles quickly overwhelms the other forces once the bubble is a sufficient size because the density difference between the air bubbles and water is so vast. It is theoretically possible to attempt to directly calculate the buoyancy force on the air bubble and correct the vertical velocity component (such that it better matches the vertical velocity of the flow). However, this requires a precise knowledge of the bubble volume and the density of the air in the bubble. Unlike water, air is highly compressible, so the density will be determined by the pressure. The pressure is trivial to estimate in relatively quiescent flows (assumed hydrostatic) but significantly less so for highly turbulent flows. Consequently, in practice it is very difficult to make corrections to the vertical velocities in this manner.

It is obviously impossible to control the size of the bubbles in a breaking wave, and undoubtedly many of the bubbles will be too large to faithfully follow the fluid motion, introducing errors in the flow velocities obtained in breaking waves by BIV (e.g., Ryu et al. 2005, 2007). In the 1: 168 scale model of a plunging breaking wave impinging on a tension-leg platform in Ryu et al. (2005), the bubbles in the breaking wave reached $5 \mathrm{~cm}$ in diameter. Even in more controlled experimental conditions, the size of the bubbles may still be a concern. In the bubble-plume, rise-velocity validation experiment presented in Ryu et al. (2005), the average size of the bubbles in the bubble plume was $3 \mathrm{~mm}$, far too large to be used as tracer particles that follow the fluid even if the particle density matched that of water perfectly. As a tacit acknowledgement of these sources of error, in most experiments involving high void fraction two-phase flows, 
BIV is performed in concert with traditional PIV, and the BIV velocities are only presented in the high void fraction regions where PIV is ineffective (e.g., Ryu et al. 2005, 2007; Ryu and Chang 2008; Chang et al. 2011). Ryu et al. (2005) directly state that the velocity obtained using BIV is "mainly the bubble velocity for the highly aerated region" and that more studies are needed to adequately describe the difference between the bubble velocity and the true flow velocity.

In this respect, acoustic cameras may be uniquely well suited to BIV. From preliminary trials with the ARIS Explorer 3000 acoustic camera equipped with the $1^{\circ}$ concentrator lens, it is very clear that the camera can easily observe bubbles that are too small to be observed with the naked eyemuch smaller than $3 \mathrm{~mm}$, though the precise size of the observed bubbles is unknown. The smaller bubbles observable by the acoustic camera will undoubtedly follow the flow better than larger bubbles, though it remains to be seen if they follow it well enough to be considered an acceptable surrogate for tracer particles. It is likely that any acoustic-camera-based BIV technique will share some of the weakness of the shadowgraphy technique (Hassan et al. 1998; Nishino et al. 2000; Lindkin and Merzkirch 2001), specifically that if the void fraction is too high, the individual bubbles may not be distinguishable, and that the acoustic scattering by extremely large bubbles may drown out that from the small (tracer) bubbles. Nonetheless, acoustic-camera-based BIV is an intriguing area of future study. 


\section{Summary}

The gaps in the existing knowledge regarding flow measurement techniques and how they may be addressed by the development of an acoustic-camera, particle-tracking-based flow measurement technique have been referred to throughout this document, but shall be summarized here for completeness. The key benefit that an acoustic-camera, particletracking-based flow measurement technique may yield is the capability to quantify flow velocity fields in turbid environments, particularly in the field. Optical-camera particle-tracking-based techniques developed to quantify velocity fields in laboratory environments, such as traditional PIV, are difficult to adapt to field studies because those techniques are equipment intensive and require stationary, well-calibrated images to be effective. Furthermore, optical-camera-based particle tracking can only be performed in a reasonably transparent fluid (such that the camera can see the light reflected by the particles). Obviously these requirements quickly become restrictive in field deployments in turbid flows.

Acoustic-based flow measurement techniques have proven very effective in collecting field measurements of flow velocities but possess their own set of limitations. Commercially available instruments such as ADVs and $\mathrm{ADCPs}$ are only able to quantify the velocity at a single point or along a transect, respectively. They are not able to quantify velocity fields and consequently cannot be used to obtain spatial gradients of velocity when deployed individually. Other acoustic-based devices have been developed to track 3D particle motion in laboratory settings, but these highly spatially and temporally resolved acoustic particle tracking systems (e.g., Mordant et al. [2005]) are only able to track a single particle (or very few particles) in a small measurement volume and thus cannot be applied to larger scale systems or used to estimate Eulerian velocity fields and spatial gradients of velocity.

Acoustic cameras may offer the capability to track a large number of particles in a plane as is required for traditional PIV and PTV flow measurement. However, only a single study (Wu et al. 2008) investigates the use of acoustic cameras for generic particle tracking, and this study did not close the gap between generic particle tracking and particle tracking required for flow measurement. In particular, Wu et al. (2008) tracked beads too large in diameter to be used as tracer particles $(8 \mathrm{~mm})$. 
Additionally, the beads were located in a fixed plane and driven by a mechanical device rather than suspended in the flow.

Several concerns will have to be addressed in the development of true acoustic-camera-based PTV or PIV for flow measurement. These are extensively discussed in Section 3.2, but in summary they involve the balance between particles big enough to be visible in the acoustic camera images and particles small enough to follow the flow. As such, a study into the development of an acoustic-camera-based PIV or PTV systems will first need to determine the smallest size tracer particle visible with the acoustic camera, as well as the tracer particle's material. Such a study would then devise an experiment to measure a simple flow (seeded with the selected tracer particles) with PIV using acoustic camera images while simultaneously measuring the flow with standard optical PIV or some other accepted flow measurement technique. Finally, the velocity vector fields yielded by the acoustic camera PIV technique could be directly compared to that from the accepted flow measurement technique for accuracy. Given that air bubbles are so easily visible in acoustic camera images, a particular subclass of PIV (i.e., BIV) is an intriguing flow measurement method in which acoustic cameras may prove particularly effective. Currently, BIV has only been used in highly turbulent and aerated flows where traditional PIV is ineffective, and the size of the air bubbles is critically important due to the drastic density difference between air and water.

In conclusion, this report describes the potential for the use of acoustic camera images in particle-tracking-based flow measurement, PTV and PIV, as well as a particular subclass of PIV (i.e., BIV). The report also describes potential considerations that will need to be addressed to develop this technique. However, a successful acoustic-camera-based PIV or BIV flow measurement system will offer the capability to quantify flow velocity fields in turbid environments. Furthermore, an acoustic-camerabased system will be substantially easier to adapt into a field-ready system due to the fact that the acoustic camera performs the primary functions of both the laser and the camera in a traditional PTV/PIV system, significantly mitigating the size and power requirements that have proven prohibitive to performing traditional PTV/PIV in field experiments. 


\section{References}

Adhikari, D., B. J. Gemmell, M. P. Hallberg, E. K. Longmire, and E. J. Buskey. 2015. "Simultaneous Measurement of 3D Zooplankton Trajectories and Surrounding Fluid Velocity Field in Complex Flows.” Journal of Experimental Biology 218(22): 3534-3540.

Adrian, R. J. 1986. "Multi-Point Optical Measurements of Simultaneous Vectors in Unsteady Flow - A Review.” International Journal of Heat and Fluid Flow 7(2): 127-145. doi:10.1016/0142-727x(86)90062-7.

Adrian, R. J. 1991. "Particle-Imaging Techniques for Experimental Fluid Mechanics." Annual Review of Fluid Mechanics 23(1): 261-304. doi:10.1146/annurev.fl.23.010191.001401.

Adrian, R. J., and J. Westerweel. 2011. Particle Image Velocimetry. Cambridge Aerospace Series No. 30. Cambridge: Cambridge University Press.

Anderson, V. C. 1950. "Sound Scattering from a Fluid Sphere." The Journal of the Acoustical Society of America 22(4): 426-431. doi:10.1121/1.1906621.

Arroyo, M. P., and C. A. Greated. 1991. "Stereoscopic Particle Image Velocimetry." Measurement Science and Technology 2(12): 1181. doi:10.1088/0957$\underline{0233 / 2 / 12 / 012 .}$

Atkinson, C., S. Coudert, J-M. Foucaut, M. Stanislas, and J. Soria. 2009. "Thick and Thin Volume Measurements of a Turbulent Boundary Layer Using Tomographic Particle Image Velocimetry." In 8th International Symposium on Particle Image Velocimetry, Melbourne, Australia.

Auton, T. R., J. C. R. Hunt, and M. Prud'Homme. 1988. "The Force Exerted on a Body in Inviscid Unsteady Non-Uniform Rotational Flow.” Journal of Fluid Mechanics 197(1): 241-257. doi:10.1017/s0022112088003246.

Belcher, E. O. 2006. "Acoustic Camera Produces Near-Video Quality Images - Dr. Edward O. Belcher (Sound Metrics Corp) Examines How ROV and Diver Crews Can Continue Working with Visual Feedback in Water Where Optical." Sea Technology 47(12): 27-31.

Belcher, E. O., B. Matsuyama, and G. M. Trimble. 2001. "Object Identification with Acoustic Lenses." OCEANS 2001, MTS/IEEE Conference and Exhibition, Vol. 1, 6-11. IEEE. doi:10.1109/oceans.2001.968656

Born, M., and E. Wolf. 1980. Principles of Optics: Electromagnetic theory of Propagation, Interference, and Diffraction of Light. $6^{\text {th }}$ ed. Oxford: Pergamon.

Candelier, F., J. R. Angilella, and M. Souhar. 2004. "On the Effect of the BoussinesqBasset Force on the Radial Migration of Stokes Particles in a Vortex." Physics of Fluids 16(5): 1765-1776. doi:10.1063/1.1689970. 
Chang, K.-A., K. Ariyarathne, and R. Mercier. 2011. "Three-Dimensional Green Water Velocity on a Model Structure." Experiments in Fluids 51(2): 327-345. doi:10.1007/s00348-011-1051-0.

Chang, K.-A., H.-J. Lim, and C. B. Su. 2003. "Fiber Optic Reflectometer for Velocity and Fraction Ratio Measurements in Multiphase Flows." Review of Scientific Instruments 74(7): 3559-3565. doi:10.1063/1.1578152.

Chao, B. T. 1964. "Turbulent Transport Behavior of Small Particles in a Turbulent Fluid." Österr Ingenieurarch, no.19: 7.

Crowe, C., M. Sommerfield, and Y. Tsuji. 1998. Multiphase Flows with Droplets and Particles. Boca Raton, FL: CRC Press.

Doron, P., L. Bertuccioli, J. Katz, and T. R. Osborn. 2001. “Turbulence Characteristics and Dissipation Estimates in the Coastal Ocean Bottom Boundary Layer from PIV Data." Journal of Physical Oceanography 31(8): 2108-2134. doi:10.1175/1520-0485(2001)031<2108:tcadei>2.0.c0;2.

Durst, F., and W. H. Stevenson. 1979. "Influence of Gaussian Beam Properties on Laser Doppler Signals.” Applied Optics 18(4): 516-524. doi:10.1364/a0.18.000516.

Elsinga, G. E., F. Scarano, B. Wieneke, and B. W. van Oudheusden. 2005. "Tomographic Particle Image Velocimetry." 6th International Symposium on Particle Image Velocimetry, Pasadena, California.

Elsinga, G. E., B. W. van Oudheusden, and F. Scarano. 2006a. "Experimental Assessment of Tomographic-PIV Accuracy." 13th International Symposium on Applications of Laser Techniques to Fluid Mechanics, Lisbon, Portugal.

Elsinga, G. E., F. Scarano, B. Wieneke, and B. W. van Oudheusden. 2006b. "Tomographic Particle Image Velocimetry." Experiments in Fluids 41(6903): 933-947.

Falkovich, G., A. Fouxon, and M. G. Stepanov. 2002. "Acceleration of Rain Initiation by Cloud Turbulence.” Nature 419(6903): 151-154. doi:10.1038/nature00983.

Faran, Jr., J. J. 1951. "Sound Scattering by Solid Cylinders and Spheres.” The Journal of the Acoustical Society of America, no. 23.4: 405-418. doi:10.1121/1.1906780.

Farazmand, M., and G. Haller. 2015. "The Maxey-Riley Equation: Existence, Uniqueness, and Regularity of Solutions." Nonlinear Analysis: Real World Applications, no. 22: 98-106. doi:10.1016/j.nonrwa.2014.08.002.

Ferrante, A., and S. E. Elghobashi. 2003. "On the Physical Mechanisms of Two-Way Coupling in Particle-Laden Isotropic Turbulence.” Physics of Fluids 15(2): 315. doi:10.1063/1.1532731.

Fritz, H. M., J. C. Borrero, C. E. Synolakis, and J. Yoo. 2006. “2004 Indian Ocean Tsunami Flow Velocity Measurements from Survivor Videos.” Geophysical Research Letters 33(24). doi:10.1029/2006GL026784.

Fritz, H. M., W. H. Hager, and H. E. Minor. 2003a. "Landslide Generated Impulse Waves." Experiments in Fluids 35(6): 505-519. doi: 10.1007/s00348-003-0660-7. 
Fritz, H. M., W. H. Hager, and H. E. Minor. 2003b. "Landslide Generated Impulse Waves. 2. Hydrodynamic Impact Craters." Experiments in Fluids 35(6): 520532. doi: $10.1007 / \mathrm{s} 00348-003-0660-7$.

Fritz, H. M., D. A. Phillips, A. Okayasu, T. Shimozono, H. Liu, F. Mohammed, V. Skanavis, C. E. Synolakis, and T. Takahashi. 2012. "The 2011 Japan Tsunami Current Velocity Measurements from Survivor Videos at Kesennuma Bay Using LiDAR." Geophysical Research Letters, no. 39(7): LooG23. doi:10.1029/2011gl050686.

Gervais, P., C. Baudet, and Y. Gagne. 2007. "Acoustic Lagrangian Velocity Measurement in a Turbulent Air Jet." Experiments in Fluids, no. 42: 371-384. http://doi:10.1007/s00348-006-0245-3http://doi:10.1007/s00348-006-02453 .

Govender, K., M. J. Alport, G. P. Mocke, and H. Michallet. 2002a. "Video Measurements of Fluid Velocity and Water Levels in Breaking Waves.” Physica Scripta, no. T97: 152-159. doi:10.1238/physica.topical.097a00152.

Govender, K., G. P. Mocke, and M. J. Alport. 2002b. "Video-Imaged Surf Zone Wave and Roller Structures and Flow Fields.” Journal of Geophysical Research: Oceans, 107(C7):9-1 - 9-21. doi:10.1029/2000jc000755.

Hassan, Y. A., W. D. Schmidl, and J. Ortiz-Villafuerte. 1998. "Investigation of ThreeDimensional Two-Phase Flow Structure in a Bubbly Pipe.” Measurement Science and Technology 9(3): 309-326. doi:10.1088/0957-0233/9/3/003.

Hecht, E., and A. Zajac. 2001. Optics. Boston, MA: Addison-Wesley Publishing Company.

Herrmann, H. J., J. S. Andrade, Jr., A. D. Aroújo, M. P. Almeida, V. Komiwes, J. Harting, and D. Kadau. 2010. "Particles in Fluids." In Complex Materials in Physics and Biology, ed. F. Mallamace and H. E. Stanley. Proceedings for the International School of Physics "Enrico Fermi" Course CLXXVI, Varenna, Italy. doi:10.3254/978-1-61499-071-0-187.

Hill, D. F., K. V. Sharp, and R. J. Adrian. 200o. "Stereoscopic Particle Image Velocimetry Measurements of the Flow around a Rushton Turbine." Experiments in Fluids 29(5): 478-485. doi:10.1007/s003480000116.

Hjemfelt, A. T., and L. F. Mockros, 1966. "Motion of Discrete Particles in a Turbulent Fluid.” Journal of Applied Sciences Research 16(1): 149-161. doi:10.1007/bf00384062.

Katija, K., S. P. Colin, J. H. Costello, and J. O. Dabiri. 2011. "Quantitatively Measuring InSitu Flows Using a Self-Contained Underwater Velocimetry Apparatus (SCUVA).” Journal of Visualized Experiments (JoVE), no. 56: e2615. https://www.ncbi.nlm.nih.gov/pmc/articles/PMC3227191/pdf/jove-56-2615.pdf.

Kim, K., N. Neretti, and N. Intrator. 2005. "Mosaicing of Acoustic Camera Images." IEEE Proceedings - Radar, Sonar and Navigation 152(4): 263-270. doi:10.1049/iprsn:20045015.

Kinsler, L., A. Frey, A. Coppens, and J. Sanders. 2000. Fundamentals of Acoustics. New York: John Wiley and Sons, Inc. 
Kolmogorov, A. N. 1941. "Dissipation of Energy in Locally Isotropic Turbulence.” Doklady Akademii Nauk SSSR 32(1): 16-18.

Kraus, N. C., A. Lohrmann, and R. Cabrera. 1994. "New Acoustic Meter for Measuring 3D Laboratory Flows." Journal of Hydraulic Engineering 120(3): 406-412. http://dx.doi.org/10.1061/(ASCE)0733-9429(1994)120:3(406).

Kundu, P. K., and I. M. Cohen. 2004. Fluid Mechanics. Cambridge, MA: Elsevier Academic Press.

Langlois, G. P., M. Farazmand, and G. Haller. 2015. "Asymptotic Dynamics of Inertial Particles with Memory.” Journal of Non-linear Science 25(6): 1225-1255. doi:10.1007/s00332-015-9250-0.

Lin, C., S.-C. Hsieh, I.-J. Lin, K.-A. Chang, and R. V. Raikar. 2012. "Flow Property and Self-Similarity in Steady Hydraulic Jumps.” Experiments in Fluids 53(5): 15911616. doi: $10.1007 / \mathrm{s} 00348-012-1377-2$.

Lindkin, R., and W. Merzkirch. 2001. "A Novel PIV Technique for Measurements in Multi-Phase Flows and Its Application to Two-Phase Bubbly Flows." Experiments in Fluids 33(6): 814-825. doi:10.1007/s00348-002-0500-1.

Lucci, F., A. Ferrante, and S. Elghobashi. 2011. "Is Stokes Number an Appropriate Indicator for Turbulence Modulation by Particles of Taylor-Length-Scale Size?" Physics of Fluids 23(2): 025101-1 - 025101-7. doi:10.1063/1.3553279.

Maas, H. G., Grün, and D. Papantoniou. 1993. "Particle Tracking in Three-Dimensional Turbulent Flows - Part 1: Photogrammetric Determination of Particle Coordinates." Experiments in Fluids 15(2): 133-146. doi:10.1007/BF00190953.

Malik, N. A., T. Dracos, and D. A. Papantoniou. 1993. "Particle Tracking Velocimetry in Three-Dimensional Turbulent Flows - Part 2: Particle Tracking." Experiments in Fluids 15(4): 279-294. doi:10.1007/BF00223406.

Maxey, M. R., and J. J. Riley. 1983. "Equations of Motion for a Small Rigid Sphere in a Non-Uniform Flow.” Physics of Fluids 26(4): 883-889. doi:10.1063/1.864230.

McFall, B. C. 2014. Physical Modeling of Landslide Generated Tsunamis in Various Scenarios from Fjords to Conical Islands. Ph.D. thesis, Georgia Institute of Technology, Atlanta, GA, USA.

Mei, R. 1996. "Velocity Fidelity of Flow Tracer Particles." Experiments in Fluids 22(1): 113. doi:10.1007/BF01893300.

Melling, A. 1997. "Tracer Particles and Seeding for Particle Image Velocimetry." Measurement Science and Technology 8(12): 1406-1416. doi:10.1088/0957$\underline{0233 / 8 / 12 / 005}$.

Melling, A., and J. H. Whitelaw. 1973. "Seeding of Gas Flows for Laser Anemometry." DISA Information, no. 15: 5-14. 
Mohammed, F., B. C. McFall, and H. M. Fritz. 2011. "Tsunami Generation by 3D Deformable Granular Landslides." Solutions to coastal disasters 2011: proceedings of the 2011 Solutions to Coastal Disasters Conference, June 26-29, 2011, Anchorage, Alaska, 310-320. Reston, VA: American Society of Civil Engineers. doi:10.1061/41185(417)28.

Mordant, N., P. Metz, O. Michel, and J. F. Pinton. 2001. "Measurement of Lagrangian Velocity in Fully Developed Turbulence.” Physical Review Letters 87(21): 214501-1 - 214501-4. doi:10.1103/physrevlett.87.214501.

Mordant, N., J. F. Pinton, and O. Michel. 2002. "Time-Resolved Tracking of a Sound Scatterer in a Complex Flow: Nonstationary Signal Analysis and Applications." The Journal of the Acoustical Society of America 112(1): 108-118. doi:10.1121/1.1477932.

Mordant, N., E. Lêvêque, and J. F. Pinton. 2004. "Experimental and Numerical Study of the Lagrangian Dynamics of High Reynolds Number Turbulence." New Journal of Physics 6(1): 116-1 - 116-44. doi:10.1088/1367-2630/6/1/116.

Mordant, N., P. Metz, O. Michel, and J. F. Pinton. 2005. "An Acoustical Technique for Lagrangian Velocity Measurements.” Review of Scientific Instruments 76(2): 025105-1 - 025105-7. http://dx.doi.org/10.1063/1.1844452.

Murphy, D. W., D. R. Webster, and J. Yen. 2012. "A High-Speed Tomographic PIV System for Measuring Zooplanktonic Flow.” Limnology and Oceanography: Methods 10(12): 1096-1112. doi:10.4319/lom.2012.10.1096.

Nishino, K., H. Kato, and K. Torii. 2000. "Stereo Imaging for Simultaneous Measurement of Size and Velocity of Particles in a Dispersed Two-Phase Flow." Measurement Science and Technology 11(6): 633-645. doi:10.1088/0957-0233/11/6/306.

Partridge, C. J. 1993. Sound Wave Scattering from a Rigid Sphere. MRL-TR-91-9. Victoria, Australia: DTSO Materials Research Laboratory. http://www.dtic.mil/cgibin/GetTRDoc?Location=U2\&doc=GetTRDoc.pdf\&AD=ADA264182.

Pedrozo-Acuña, A., A. R. de Alegría-Arzaburu, A. Torres-Freyermuth, E. Mendoza, and R. Silva. 2011. "Laboratory Investigation of Pressure Gradients Induced by Plunging Breakers." Coastal Engineering 58(8): 722-738. doi:10.1016/i.coastaleng.2011.03.013.

Pope, S. B. 2000. Turbulent Flows. Cambridge: Cambridge University Press.

Prasad, A. K., and R. J. Adrian. 1993. "Stereoscopic Particle Image Velocimetry Applied to Liquid Flows.” Experiments in Fluids 15(1): 49-60. doi:10.1007/BF00195595.

Prasad, A. K. 2000. "Stereoscopic Particle Image Velocimetry." Experiments in Fluids 29(2): 103-116. doi:10.1007/s003480000143.

Qureshi, N. M., M. Bourgoin, C. Baudet, A. Cartellier, and Y. Gagne. 2007. "Turbulent Transport of Material Particles: An Experimental Study of Finite Size Effects." Physical Review Letters 99(18): 184502-1 - 184502-4. doi:10.1103/physrevlett.99.184502. 
Qureshi, N. M., U. Arrieta, C. Baudet, A. Cartellier, Y. Gagne, and M. Bourgoin. 2008. "Acceleration Statistics of Inertial Particles in Turbulent Flow." European Physical Journal B 66(4): 531-536. doi:10.1140/epjb/e2008-00460-x.

Raffel, M., C. Willert, S. Wereley, and J. Kompenhans. 2007. Particle Image Velocimetry: A Practical Guide, $2^{\text {nd }}$ edition. Berlin: Springer-Verlag.

Ryu, Y., and K.-A. Chang. 2008. "Green Water Void Fraction Due to Breaking Wave Impinging and Overtopping.” Experiments in Fluids 45(5): 883-898.

Ryu, Y., K.-A. Chang, and H. J. Lim. 2005. "Use of Bubble Image Velocimetry for Measurement of Plunging Wave Impinging on a Structure and Associated Greenwater." Measurement Science and Technology 16(10): 1945-1953. http://dx.doi.org/10.1088/0957-0233/16/10/009.

Ryu, Y., K.-A. Chang, and R. Mercier. 2007. "Runup and Green Water Velocities Due to Breaking Wave Impinging and Overtopping." Experiments in Fluids 43(4): 555567. doi:10.1007/s00348-007-0332-0.

Scarano, F. 2013. "Tomographic PIV: Principles and Practice." Measurement Science and Technology 24(1): 012001. doi:10.1088/0957-0233/24/1/012001.

Shaw, R. A., and S. P. Oncley. 2001. "Acceleration Intermittency and Enhanced Collision Kernels in Turbulent Clouds." Atmospheric Research 59(60): 77-87. doi:10.1016/s0169-8095(01)00110-7.

Smith, S. J., and C. T. Friedrichs. 2015. "Image Processing Methods for In Situ Estimation of Cohesive Sediment Floc Size, Settling Velocity, and Density." Limnology and Oceanography: Methods, no. 13: 250-264. doi:10.1002/lom3.10022.

Smith, W. N., P. Atsavapranee, J. Katz, and T. Osborn. 2002. "PIV Measurements in the Bottom Boundary Layer of the Coastal Ocean." Experiments in Fluids 33(6): 962-971. doi:10.1007/s00348-002-0490-z.

Soloff, S. M., R. J. Adrian, and Z-C. Liu. 1997. "Distortion Compensation for Generalized Stereoscopic Particle Image Velocimetry.” Measurement Science and Technology 8(12): 1441-1454. doi:10.1088/0957-0233/8/12/008.

Stanton, T. K., P. H. Wiebe, and D. Chu. 1998. "Differences between Sound Scattering by Weakly Scattering Spheres and Finite-Length Cylinders with Applications to Sound Scattering Zooplankton.” The Journal of the Acoustical Society of America 103(1): 254-264. doi:10.1121/1.421135.

Takeda, Y. 1986. "Velocity Profile Measurement by Ultrasound Doppler Shift Method." International Journal of Heat and Fluid Flow 7(4): 313-318. doi:10.1016/0142$\underline{727 x(86) 90011-1 .}$.

Tennekes, H., and J. L. Lumley. 1972. A First Course in Turbulence. Cambridge, MA: MIT Press.

Thorne, P. D., and D. M. Hanes. 2002. "A Review of Acoustic Measurements of SmallScale Sediment Processes.” Continental Shelf Research 22(4): 603-632. doi:10.1016/s0278-4343(01)00101-7. 
Tropea, C., A. Yarin, and J. F. Foss. 2007. Springer Handbook of Experimental Fluid Mechanics. Berlin: Springer-Verlag.

Urick, R. J. 1983. Principles of Underwater Sound. $3^{\text {rd }}$ ed. New York: McGraw-Hill.

van Doorne, C. W. H., and J. Westerweel. 2007. "Measurement of Laminar, Transitional and Turbulent Pipe Flow Using Stereoscopic-PIV." Experiments in Fluids 42(2): 259-279. doi:10.1007/s00348-006-0235-5.

van Veen, B. D., and K. M. Buckley. 1988. "Beamforming: A Versatile Approach to Spatial Filtering.” IEEE ASSP Magazine 5(2): 4-24. doi:10.1109/53.665.

Voulgaris, G., and J. H. Trowbridge. 1998. "Evaluation of the Acoustic Doppler Velocimeter (ADV) for Turbulence Measurements." Journal of Atmospheric and Oceanic Technology 15(1): 272-289. doi:10.1175/1520-

0426(1998)015<0272:EOTADV>2.0.C0;2.

Webster, D. R., D. L. Young, and J. Yen. 2015. “Copepods Response to Burgers' Vortex: Deconstructing Interactions of Copepods with Turbulence." Integrative and Comparative Biology 55 (4): 706-718. doi:10.1093/icb/icv054.

Webster, D. R., and D. L. Young. 2015. “A Laboratory Realization of the Burgers' Vortex Cartoon of Turbulence-Plankton Interactions." Limnology and Oceanography: Methods 13(2): 92-102. doi:10.1093/icb/icv054.

Weill, A. 2014. "Acoustic Radiation.” In Encyclopedia of Remote Sensing, 1-4. New York: Springer. doi:10.1007/978-0-387-36699-9_1.

Westerweel, J., and J. van Oord. 2000. "Stereoscopic PIV Measurements in a Turbulent Boundary Layer.” In Particle Image Velocimetry: Progress towards Industrial Application, ed. M. Stanislaus, J. Kompenhans, and J. Westerweel, 459-478. Dordrecht: Kluwer Academic Publishers.

Westerweel, J. 1993. Digital Particle Image Velocimetry - Theory and Application. Ph.D. dissertation, Delft University Press, Delft.

Wieneke, B. 2008. "Volume Self-Calibration for 3D Particle Image Velocimetry." Experiments in Fluids 45(4): 549-556. doi:10.1007/s00348-008-0521-5.

Wu, F.-C., Y.-C. Shao, C.-K. Wang, and J. Liou. 2008. "Potential for Application of an Acoustic Camera in Particle Tracking Velocimetry." Review of Scientific Instruments 79(11): 116102-1 - 116102-3. http://dx.doi.org/10.1063/1.3021046.

Yang, T. S., and S. S. Shy. 2005. "Two-Way Interaction between Solid Particles and Homogeneous Air Turbulence: Particle Settling Rate and Turbulence Modification Measurements.” Journal of Fluid Mechanics, no. 526: 171-216. doi:10.1017/s0022112004002861.

Young, D. L., B. L. Bruder, K. A. Haas, and D. R. Webster. 2016. "The Hydrodynamics of Surface Tidal Flow Exchange in Saltmarshes." Estuarine, Coastal and Shelf Science, no. 172: 128-137. doi:10.1016/i.ecss.2016.02.006.

Young, D. L. 2014. Turbulence-Copepod Interaction: Acartia tonsa Behavioral Response to Burgers' Vortex. M.S. thesis, Georgia Institute of Technology, Atlanta, GA, USA. 


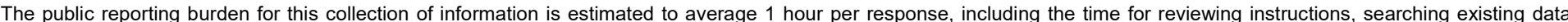

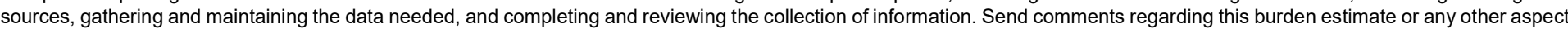

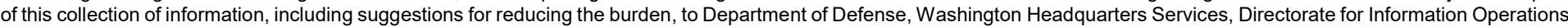

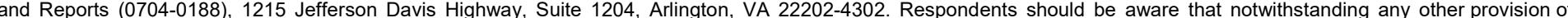
law, no person shall be subject to any penalty for failing to comply with a collection of information if it does not display a currently valid OMB control number.

PLEASE DO NOT RETURN YOUR FORM TO THE ABOVE ADDRESS.

\begin{tabular}{l|l|l|}
\hline $\begin{array}{l}\text { 1. REPORT DATE } \\
\text { August } 2017\end{array}$ & $\begin{array}{l}\text { 2. REPORT TYPE } \\
\text { Final Report }\end{array}$ & 3. DATES COVERED (From - To)
\end{tabular}

\section{TITLE AND SUBTITLE}

The Feasibility of Performing Particle-Tracking-Based Flow Measurements with Acoustic Cameras 5a. CONTRACT NUMBER

5b. GRANT NUMBER

5c. PROGRAM ELEMENT NUMBER

5d. PROJECT NUMBER

DOER 17-02

5e. TASK NUMBER

5f. WORK UNIT NUMBER

8. PERFORMING ORGANIZATION REPORT NUMBER

ERDC/CHL SR-17-1

Coastal and Hydraulics Laboratory

U.S. Army Engineer Research and Development Center

3909 Halls Ferry Road

Vicksburg, MS 39180-6199

9. SPONSORING/MONITORING AGENCY NAME(S) AND ADDRESS(ES)

U.S. Army Corps of Engineers

Washington, DC 20314-1000
10. SPONSOR/MONITOR'S ACRONYM(S) HQUSACE

11. SPONSOR/MONITOR'S REPORT NUMBER(S)

\section{DISTRIBUTION/AVAILABILITY STATEMENT}

Approved for public release; distribution is unlimited.

\section{SUPPLEMENTARY NOTES}

\section{ABSTRACT}

Modern science lacks the capability to quantify flow velocity fields in turbid environments, particularly in the field. Existing opticalcamera particle-tracking-based techniques developed to quantify two-dimensional (2D) velocity fields in laboratory environments, such as particle image velocimetry (PIV), are equipment intensive and can only be performed in a reasonably transparent fluid (so the camera can observe the light reflected by the particles). Acoustic-based flow measurement equipment used in the field (e.g., acoustic Doppler velocimeter and acoustic Doppler current profiler) can readily acquire velocities in turbid flows but only measure point velocities or velocities along a transect. Consequently, they do not provide the capability to quantify spatial gradients of velocity when deployed individually. The objective of this report is to explore whether acoustic cameras can be used in place of optical cameras in particletracking-based flow measurement techniques, such as PIV. Acoustic cameras may offer the capability to track a large number of particles in a plane as is required for traditional PIV and are designed to operate in turbid environments. The development of an acoustic camera particle-tracking-based flow measurement system will be able to quantify $2 \mathrm{D}$ flow velocity fields in turbid environments and be readily adaptable to a field-based system.

\section{SUBJECT TERMS}

Acoustic velocity meters, Flow meters, Fluid dynamics, Scientific apparatus and instruments--Evaluation

\section{SECURITY CLASSIFICATION OF:}

\begin{tabular}{|l|c|c|c|} 
a. REPORT & b. ABSTRACT & c. THIS PAGE & ABSTRACT \\
Unclassified & Unclassified & Unclassified & SAR \\
& & & \\
\end{tabular}

18. NUMBER OF PAGES

51 19a. NAME OF RESPONSIBLE PERSON

David L. Young

19b. TELEPHONE NUMBER (Include area code) $252.261 .6840 \times 225$ 